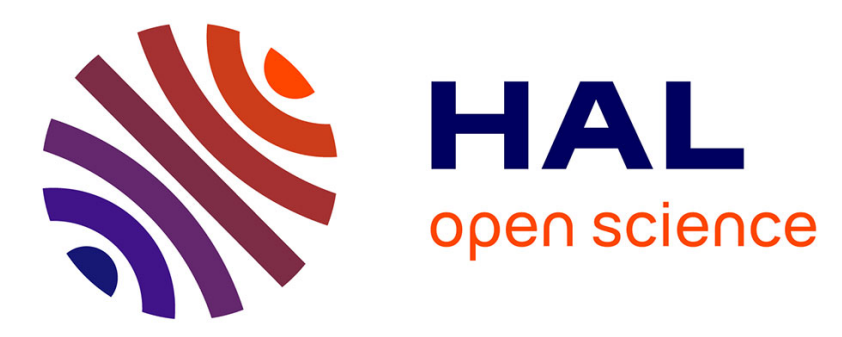

\title{
Low-frequency sound radiated by a nonlinearly modulated wavepacket of helical modes on a subsonic circular jet
}

Xuesong $\mathrm{Wu}$, Patrick Huerre

\section{- To cite this version:}

Xuesong $\mathrm{Wu}$, Patrick Huerre. Low-frequency sound radiated by a nonlinearly modulated wavepacket of helical modes on a subsonic circular jet. Journal of Fluid Mechanics, 2009, 637, pp.173-211. $10.1017 / \mathrm{s} 0022112009990577$. hal-01002596

HAL Id: hal-01002596

https://hal-polytechnique.archives-ouvertes.fr/hal-01002596

Submitted on 7 Jul 2014

HAL is a multi-disciplinary open access archive for the deposit and dissemination of scientific research documents, whether they are published or not. The documents may come from teaching and research institutions in France or abroad, or from public or private research centers.
L'archive ouverte pluridisciplinaire HAL, est destinée au dépôt et à la diffusion de documents scientifiques de niveau recherche, publiés ou non, émanant des établissements d'enseignement et de recherche français ou étrangers, des laboratoires publics ou privés. 


\title{
Low-frequency sound radiated by a nonlinearly modulated wavepacket of helical modes on a subsonic circular jet
}

\author{
XUESONG WU ${ }^{1,2}$ AND PATRICK HUERRE \\ ${ }^{1}$ Department of Mathematics, Imperial College London, 180 Queen's Gate, London SW7 2AZ, UK \\ ${ }^{2}$ Department of Mechanics, Tianjin University, Tianjin 300072, People's Republic of China \\ ${ }^{3}$ Laboratoire d'Hydrodynamique (LadHyX) CNRS-Ecole Polytechnique, \\ F-91128 Palaiseau, France
}

(Received 3 June 2008; revised 18 May 2009; accepted 21 May 2009; first published online

23 September 2009)

A possible fundamental physical mechanism by which instability modes generate sound waves in subsonic jets is presented in the present paper. It involves a wavepacket of a pair of helical instability modes with nearly the same frequencies but opposite azimuthal wavenumbers. As the wavepacket undergoes simultaneous spatial-temporal development in a circular jet, the mutual interaction between the helical modes generates a strong three-dimensional, slowly modulating 'mean-flow distortion'. It is demonstrated that this 'mean field' radiates sound waves to the far field. The emitted sound is of very low frequency, with characteristic time and length scales being comparable with those of the envelope of the wavepacket, which acts as a noncompact source. A matched-asymptotic-expansion approach is used to determine, in a self-consistent manner, the acoustic field in terms of the envelope of the wavepacket and a transfer factor characterizing the refraction effect of the background base flow. For realistic jet spreading rates, the nonlinear development of the wavepacket is found to be influenced simultaneously by non-parallelism and non-equilibrium effects, and so a composite modulation equation including both effects is constructed in order to describe the entire growth-attenuation-decay cycle. Parametric studies pertaining to relevant experimental conditions indicate that the acoustic field is characterized by a single-lobed directivity pattern beamed at an angle about $45^{\circ}-60^{\circ}$ to the jet axis and a broadband spectrum centred at a Strouhal number $S t \approx 0.07-0.2$. As the nonlinear effect increases, the radiation becomes more efficient and the noise spectrum broadens, but the gross features of the acoustic field remain robust, and are broadly in agreement with experimental observations.

\section{Introduction}

The idea that instability waves in transitional flows, or, more broadly, large-scale coherent structures in fully turbulent flows, could be an important source of noise has attracted a great deal of interest. It was first proposed by Bishop, Ffowcs Williams \& Smith (1971) and Tam (1971), among others, in order to explain some distinctive features of supersonic jet noise. Following the discovery of orderly structures in subsonic jets (Bradshaw, Ferriss \& Johnson 1964; Crow \& Champagne 1971), it 
was soon suggested that these structures might be related to sound emission in the subsonic regime as well (Crow 1972).

The physical mechanisms by which instability modes radiate sound are by no means obvious. The reason is that shear flows (e.g. jets and boundary layers) act as waveguides so that instability modes propagate primarily in the streamwise direction, while in the transverse direction they attenuate rapidly. The energy carried by instability waves thus remains 'trapped' in the shear layer, with little or no radiation into the far field.

In supersonic flows such as jets or mixing layers, there exist the so-called supersonic instability modes when the free-stream Mach number exceeds unity by a sufficient amount. The propagation velocity of these modes relative to the free stream is greater than the ambient sound speed. In the majority of the flow field, the eigenfunction of a supersonic mode decays exponentially in the transverse direction. However, in the vicinity of its neutral position, the eigenfunction remains finite or decays algebraically, and part of its energy radiates into the far field in the form of Mach waves. This mechanism can easily be understood in terms of the 'wavy-wall analogy', which explains the 'phase' propagation of the radiated Mach waves (Tam 1995). In reality, however, instability waves are usually modulated in space rather than purely sinusoidal, and the radiated Mach waves then appear as beams, a feature that cannot be explained by the usual wavy-wall analogy. Tam \& Burton (1984) formulated an asymptotic theory to calculate the sound emitted by a linearly evolving supersonic mode. Wu (2005) recently considered Mach-wave radiation by nonlinearly evolving supersonic waves or wavetrains. By taking advantage of the fact that the time and length scales of the phase and envelope of a Mach wave are asymptotically distinct, Wu was able to express the acoustic field explicitly in terms of the amplitude of the instability mode. This solution represents an 'extended wavy-wall analogy', as it shows how the 'envelope' of a wavepacket propagates to form a distinct Mach-wave beam. In the high-Mach-number regime in which supersonic modes are dominant, instability waves may indeed act as a dominant source of noise, and Mach-wave radiation by supersonically propagating large-scale structures, as a fundamental mechanism, underpins much of our current understanding of noise generation by turbulent jets (see the review by Tam 1995).

Almost all commercial aircraft currently in service however operate in the subsonic or moderate supersonic regime, in which relevant instability modes are subsonic; i.e. they propagate subsonically relative to the ambient stream. Their role in noise generation remains a topic of debate. The eigenfunction of a subsonic mode evolving linearly in a parallel flow exhibits exponential decay in the transverse direction everywhere, including at the neutral position, and a purely sinusoidal mode thus emits no sound wave. However, under the combined effects of nonlinearity and mean-flow spreading, instability waves actually undergo amplification followed by saturation and decay over a long length scale. Such a spatially modulated wave contains a supersonic Fourier component and hence emits a sound wave with the same frequency as that of the instability mode. This mechanism, which may be termed 'direct radiation', was demonstrated by Tam \& Morris (1980) in the case of a subsonic jet and by Crighton \& Huerre (1990) in a somewhat abstract setting. The latter work revealed some delicate dependence of the radiated sound on the envelope shape of the instability wave. Acoustic emission of wavepackets modulated in both time and space was analysed by Akylas \& Toplosky (1986) and Haj-Hariri \& Akylas (1986).

The intensity of the sound waves generated via the direct-radiation mechanism by a subsonic mode modulated over a length scale much longer than its wavelength 
is typically exponentially small (with respect to the ratio of these scales). Strong radiation occurs when a wavetrain experiences a rapid change in the sense that its amplitude varies over a length scale comparable with its own wavelength. Abrupt adjustments of this kind often arise due to externally imposed geometric features, such as sharp edges (e.g. the trailing edge of an aerofoil) and isolated surface roughness. As an instability mode propagates through such a region, part of its energy is scattered into sound waves. Such a sound generation process has been investigated theoretically by Wu \& Hogg (2006) using a model problem, where a Tollmien-Schlichting wave interacts with a local surface roughness.

Abrupt changes may also occur due to the intrinsic dynamics of the flow. For jets and mixing layers, a particular case of interest is vortex pairing, which causes a sudden thickening of the local shear-layer width. Emission of sound from this process has been studied experimentally (Kibens 1980; Laufer \& Yen 1983; Bridges \& Hussain 1992) and by means of direct numerical simulation (DNS; e.g. Mitchell, Lele \& Moin 1999). A theoretical model was proposed by Goldstein (1984), but this calculation suggests the emission is not strong enough to explain the experimental findings.

Experimental studies conducted since the early 1960s have accumulated a vast collection of data for subsonic jet noise (e.g. Mollo-Christensen, Kolpin \& Martuccelli 1964; Lush 1971; Ahuja et al. 1982; Bogey et al. 2007). In the following, we highlight some of the key features which cannot be explained by existing mechanisms. The most striking one is the disparity between the time scales of the dominant hydrodynamic and acoustic fluctuations within the jet and in the far field respectively. The spectral peak of the far-field noise is centred at Strouhal number $S t \equiv f^{*} D / U_{J} \approx 0.2$, where $f^{*}$ is the dimensional frequency and $D$ and $U$ denote the nozzle diameter and exit velocity respectively. The peak frequency of hydrodynamic fluctuations in the noise production region, which may extend up to 20 diameters from the jet nozzle up to the end of the potential core, is a decreasing function of the downstream distance: the corresponding characteristic Strouhal number decreases continuously from $S t \approx 4$ near the nozzle to $S t \approx 0.5$ towards the end of potential core. This disparity of the time scales suggests that the most energetic fluctuations within the jet do not 'directly' emit dominant noise. Generation of noise must involve an 'inverse-cascade' process, through which the energy in relatively high-frequency components is transferred to low-frequency ones before being radiated in the form of sound. The overall noise, while less directional than the supersonic counterpart, appears to concentrate along the direction that makes an angle about $30^{\circ}$ to the jet axis.

A particularly interesting and curious behaviour of subsonic jets is 'noise amplification' by tonal excitation, a phenomenon in which exciting a jet at a pure tone leads to significant enhancement of broadband far-field noise. This was first observed by Bechert \& Pfizenmaier (1975) and Moore (1979) for axisymmetric modes. Subsequently, Bechert \& Pfizenmaier (1977) and Ahuja et al. (1982) found that exciting helical modes had a similar effect. Further investigations (Hussain \& Hasan 1985; Zaman 1985) showed that exciting a relatively high-frequency shear-layer mode may 'suppress' broadband noise instead.

Considerable efforts have been devoted to identifying and characterizing vortical structures which might be relevant for noise generation. Large-scale, coherent structures were found to dominate the noise-producing region in both natural unexcited (Bradshaw et al. 1964; Moore 1977; Zaman \& Hussain 1984) and excited (Crow \& Champagne 1971; Chan 1974; Zaman \& Hussain 1980) jets. They exhibit the characteristics of wavepackets undergoing amplification, saturation and decay in the axial direction. A remarkable fact is that even though the jet is fully turbulent, 
the wavelengths, propagation speeds and radial distributions of these structures are quite well described by local linear instability modes supported by the background mean flow (e.g. Michalke 1971; Suzuki \& Colonius 2006). Their axial development is significantly influenced by nonlinear effects in typical laboratory and practical conditions; only the evolution of a small-amplitude disturbance may be predicted approximately by a linear non-parallel theory, which accounts for the slow divergence of the jet (Crighton \& Gaster 1976).

Recently, proper orthogonal decomposition (POD) has been applied to extract dominant structures from experimental data. By performing POD analysis of the hydrodynamic pressure signal measured at the outer edge of the shear layer, Arndt, Long \& Glauser (1997) found axisymmetric and helical modes with azimuthal wavenumber $m= \pm 1$ to be the most energetic components in the near field. Among these, high-frequency components saturate earlier than low-frequency ones, and as a result, the frequency of the dominant structure decreases in the axial direction. The POD analysis of the streamwise velocity showed that in addition to the axisymmetric and first helical modes, modes with $m$ up to \pm 6 are present in the near field (Citriniti \& George 2000; Jung, Gamard \& George 2004; Iqbal \& Thomas 2007). The axisymmetric mode and helical modes with $|m|>2$ gradually diminish as $x$ increases, while the first helical modes $(m= \pm 1)$ remain dominant. These results are fully consistent with the linear stability properties of axisymmetric jets (cf. Strange \& Crighton 1983; Cohen \& Wygnanski 1987). Indeed, the temporal and spatial properties of the extracted POD modes closely resemble those of inviscid unstable modes, although a single POD mode does not always represent the entire evolution of an instability wave (Suzuki \& Colonius 2006). The most interesting finding is that beyond the end of the potential core, near-zero-frequency modes with $m= \pm 2$ and $m=0$ acquire significant amplitudes (Gamard, Jung \& George 2004). We believe that these components are not linear eigenmodes; rather they represent the 'mean-flow distortion' driven by the nonlinear interaction between two slightly detuned helical $m= \pm 1$ modes. Such a nonlinear generation of exceptionally large azimuthally dependent mean flow was observed experimentally in laminar transitional jets long time ago (Cohen \& Wygnanski 1987b; Long \& Petersen 1992). Gamard et al. (2004) also cited some evidence which suggests that the near-zero-frequency $m= \pm 2$ modes may be linked to noise generation. Prompted by the above observations, in the present paper we shall develop a mathematical theory to demonstrate how the $m= \pm 2$ modes are produced by nonlinear interactions and, more importantly, how they emit sound.

Measurements of both hydrodynamic motions within the jet and the far-field acoustics have been made in order to seek a cause-effect relation. The experiment of Stromberg, Mclaughlin \& Troutt (1980) for a Mach number 0.9 jet at a low Reynolds number is of particular interest because a laminar shear layer exists close to the nozzle, where the fluctuations consist of a narrow band of instability waves with frequencies centred at $S t=0.44$. The acoustic field, however, has a broadband spectrum with a peak at $S t \approx 0.22$, which led the authors to suggest that the noise was generated through vortex pairing. DNS pertaining to this low-Reynolds-number condition (Freund 2001) shows that the peak radiating component of the source coincides with neither the peak of the fully unfiltered source nor that of the kinetic energy. Filtering out the non-radiating components of the full source at a single frequency reveals the relevant source to be a non-compact, modulated wavepacket. Measurements in a high-Reynolds-number turbulent jet (Zaman 1986) indicate that the noise source of a given frequency is located at the position at which the 
axial-velocity spectral component at that frequency is maximum, but this does not mean that the strongest fluctuation radiates the strongest sound. Hileman et al. (2005) sought for the relation between coherent structures and noise generation in the time domain, by detecting and comparing distinctive features of hydrodynamic events during the relatively short noise generation and the prolonged quiet periods. Their study lends further support to the earlier suggestion by Morrison \& McLaughlin (1979) that dominant noise is emitted when coherent structures disintegrate and break down into small-scale motions.

Extensive research efforts in the past 40 years have provided an abundant collection of data about the characteristics of subsonic jet noise and the dynamics of large-scale structures and have thereby significantly improved our understanding of the relation between them. However, much of this understanding stays at the level of intuitive phenomenological descriptions. The assertion by Zaman (1986) that 'the precise noise production mechanism in terms of [the large-scale structure] dynamics still remains largely unknown' holds true to the present day. It may be stressed that understanding this mechanism is especially important. This is because these quasi-deterministic structures are known to be sensitive to ambient (naturally present or artificially excited for control purpose) perturbations and therefore are unlikely to possess the statistical universality implied in current popular engineering methodologies based on unsteady Reynolds-averaged Navier-Stokes equations and acoustic analogy (e.g. Khavaran \& Bridges 2004). An improved prediction scheme may emerge if the noise produced by these structures can be accounted for separately in a framework based on their quasi-deterministic nature and the underlying physical mechanism of radiation.

In this paper, we shall describe a new physical mechanism by which an instability wavepacket generates sound. A high-Reynolds-number asymptotic approach will be taken to describe the nonlinear evolution of the wavepacket and to analyse and predict the acoustic radiation on the basis of first principles. Strictly speaking, the formulation is for a laminar jet undergoing transition, but given the connection between coherent structures in turbulent flows and instability waves as presented above, the basic mechanism identified in this paper is expected to operate in turbulent jets. On the basis of this, theoretical predictions will be related to experimental results for turbulent jets. Alternatively, one might view the present analysis as being applied to the averaged profile of a turbulent flow, with the direct influence of small-scale turbulence (i.e. fluctuations which are on scales much smaller than the integral length scale of the turbulence) on coherent structures being neglected; this issue will be discussed further at the end of this paper.

The rest of the paper is organized as follows. In $\S 2$, the problem is formulated. The disturbance is assumed to be a wavepacket consisting of a pair of helical modes, which is modulated simultaneously in both time and space on an axisymmetric jet. Its nonlinear development in the non-equilibrium critical-layer regime is considered first in $\S 3.1$. The evolution equation for the amplitude function is deduced, by a minor modification, from the one given by $\mathrm{Wu}$, Lee \& Cowley (1993) for oblique modes in a plane shear layer. The nonlinear interaction between the helical modes within the critical layer generates a mean-flow distortion, which is modulated in space but also slowly 'breathes' in time, as will be shown in $\S 3.2$. By analysing the asymptotic behaviour of this spatially and temporally modulated 'mean field' far away from the jet axis, we show that it emits low-frequency sound waves. The characteristics of these waves are determined by an asymptotic approach in $\S 3.3$. In $\S 4$, we consider the evolution in the equilibrium critical-layer regime in which the non-parallel effect is included. Finally, a composite amplitude equation accounting for 
non-equilibrium, nonlinearity and non-parallelism is formed. Results of parametric studies are presented in $\S 5$. A summary and concluding remarks are given in $\S 6$.

\section{Formulation}

We consider a circular jet, for which it is natural to use cylindric polar coordinates $(x, r, \phi)$. The velocity components in the axial, radial and azimuthal directions are denoted by $(u, v, w)$. The nozzle radius $R_{J}$ and the exit speed $U_{J}$ will be taken as reference length and velocity so that the reference time is $R_{J} / U_{J}$. The density $\rho$ (temperature $\theta$ ) is non-dimensionalized by the jet density $\rho_{J}$ (temperature $\Theta_{J}$ ) and the pressure $p$ by $\rho_{J} U_{J}^{2}$. The Mach number and Reynolds number are defined as

$$
M=U_{J} / c_{J}, \quad R=U_{J} R_{J} / \nu_{J},
$$

where $c_{J}$ is the sound speed and $v_{J}$ the kinematic viscosity at the jet exit. Note that the present $R$ is half the usual Reynolds number $R_{D}$ based on the diameter $D$. We assume that $M=O(1)$ and $R$ is asymptotically large, i.e. $R \gg 1$. The velocity profile of the shear flow is given by

$$
\left(U\left(x_{3}, r\right), R^{-1} V\left(x_{3}, r\right)\right) \quad \text { with } \quad x_{3}=x / R .
$$

The disturbance of interest is a wavepacket consisting of a pair of helical modes with nearly the same streamwise wavenumber $\alpha$ and frequency $\omega_{c}$ but opposite azimuthal wavenumbers $\pm m$. As the disturbance propagates downstream, it will amplify until approaching its neutral position, say $x_{n}$, after which it starts to decay if its amplitude is sufficiently small. However, if a certain threshold order of magnitude is reached before approaching $x_{n}$, the disturbance then evolves into a nonlinear stage because of the emergence of a critical layer, i.e. a thin region surrounding the level at which the velocity of the basic flow equals the phase speed of the instability modes. Nonlinearity becomes significant in this layer because the disturbance attains its largest amplitude. The continued development can be described by nonlinear critical-layer theory. The reader is referred to Goldstein (1995) and Cowley \& Wu (1995) for reviews.

Depending on the initial amplitude, two nonlinear regimes are possible in principle, the so-called non-equilibrium parallel regime and the equilibrium non-parallel regime. These will be considered in turn. Eventually, a composite solution will be constructed to unify both regimes.

\section{The non-equilibrium critical-layer regime}

\subsection{Instability modes}

The nonlinear evolution of a pair of interacting helical modes on a circular jet is similar to that of a pair of oblique modes in a planar shear layer, considered by Goldstein \& Choi (1989) and Wu et al. (1993). The nonlinear regime that the helical modes can enter depends on $\epsilon$, their magnitude in the main part of shear flow. When $\epsilon \sim R^{-1}$, the nonlinear interaction occurs in the so-called non-equilibrium viscous critical-layer regime, taking place at

$$
x_{3} \approx x_{n}+R^{-1 / 3} \Delta,
$$

where $\Delta=O(1)$. The growth rate has diminished to $O\left(R^{-1 / 3}\right)$ so that the appropriate slow space and time variables to describe the ensuing nonlinear evolution are

$$
\tilde{x}=R^{-1 / 3}\left(x-x_{0}\right) \equiv R^{2 / 3}\left(x_{3}-\left(x_{n}+R^{-1 / 3} \Delta\right)\right), \quad \tilde{t}=R^{-1 / 3} t,
$$


where the origin of $\tilde{x}$ is shifted in order to facilitate the discussion of non-parallelism later. In view of the threshold amplitude stated earlier, we may set

$$
\epsilon=R^{-1} \text {. }
$$

The velocity and temperature profiles of the base flow can be approximated, to the required order, by

$$
\left(\bar{U}\left(r, x_{3}\right), \bar{T}\left(r, x_{3}\right)\right) \approx\left(\bar{U}\left(r, x_{n}\right), \bar{T}\left(r, x_{n}\right)\right)+R^{-1 / 3}\left(\bar{U}_{1}(r), \bar{T}_{1}(r)\right)\left(\Delta+R^{-1 / 3} \tilde{x}\right) .
$$

In the following, unless otherwise stated, $\bar{U}$ and $\bar{T}$ are to be understood to stand for $\bar{U}\left(r, x_{n}\right)$ and $\bar{T}\left(r, x_{n}\right)$ respectively.

In the main part of the jet, the disturbance expands as (cf. Wu et al. 1993)

$$
\begin{aligned}
(u, v, w, p, \theta, \rho)= & \epsilon \tilde{A}(\tilde{x}, \tilde{t})\left(u_{0}, v_{0}, w_{0}, p_{0}, \theta_{0}, \rho_{0}\right) E \cos m \phi \\
& +\epsilon\left(u_{m}, R^{-1 / 3} v_{m}, R^{-1 / 3} w_{m}, R^{-2 / 3} p_{m}, \theta_{m}, \rho_{m}\right) \cos (2 m \phi) \\
& +\epsilon R^{-1 / 3}\left(u_{1}, v_{1}, w_{1}, p_{1}, \theta_{1}, \rho_{1}\right) E \cos m \phi+\text { c.c. }+\ldots
\end{aligned}
$$

here $\epsilon$ measures the magnitude of the helical modes, which for simplicity are assumed to have a common envelope function $\tilde{A}(\tilde{x}, \tilde{t})$. The dependence on suitable slow space and time variables, $\tilde{x}$ and $\tilde{t}$, is introduced to account for simultaneous temporalspatial modulation, which is crucial for acoustic radiation. For convenience, we have set

$$
E=\mathrm{e}^{\mathrm{i}\left(\alpha x-\omega_{c} t\right)}
$$

In expansion (3.3), the variables with subscripts 0,1 and $m$ represent, respectively, the eigenfunction of the neutral helical modes, the deviation of the disturbance from the neutrality and the nonlinearly generated slowly modulated mean-flow distortion. The disparity between the scales of the carrier waves and their envelope determines the order-of-magnitude relation in expansion (3.3).

The governing equations, at leading order, are

$$
\begin{gathered}
\mathrm{i} \alpha(\bar{U}-c) \rho_{0}+\bar{R}^{\prime} v_{0}+\bar{R}\left(\mathrm{i} \alpha u_{0}+\frac{\partial v_{0}}{\partial r}+\frac{v_{0}}{r}+\frac{m}{r} w_{0}\right)=0 \\
\mathrm{i} \alpha(\bar{U}-c) u_{0}+\bar{U}^{\prime} v_{0}=-\mathrm{i} \alpha \bar{T} p_{0} \\
\mathrm{i} \alpha(\bar{U}-c) v_{0}=-\bar{T} \frac{\partial p_{0}}{\partial r} \\
\mathrm{i} \alpha(\bar{U}-c) w_{0}=\frac{\bar{T} m}{r} p_{0} \\
\mathrm{i} \alpha(\bar{U}-c) \theta_{0}+\bar{T}^{\prime} v_{0}=-\mathrm{i} \alpha M^{2}(\gamma-1)(\bar{U}-c) p_{0}
\end{gathered}
$$

where $c=\omega_{c} / \alpha$ is the phase speed. On eliminating $u_{0}, v_{0}, w_{0}, \rho_{0}$ and $\theta_{0}$ in favour of the pressure, we obtain

$$
\left\{\frac{\partial^{2}}{\partial r^{2}}+\frac{1}{r} \frac{\partial}{\partial r}+\left(\frac{\bar{T}^{\prime}}{\bar{T}}-\frac{2 \bar{U}^{\prime}}{\bar{U}-c}\right) \frac{\partial}{\partial r}+\left(\frac{\alpha^{2} M^{2}}{\bar{T}}(\bar{U}-c)^{2}-\alpha^{2}-\frac{m^{2}}{r^{2}}\right)\right\} p_{0}=0 .
$$

In the vicinity of the critical level $r_{c}$, where $\bar{U}\left(r_{c}\right)-c=0, p_{0}$ has the local asymptotic solution (cf. Leib 1991; Wu 2005)

$$
p_{0} \sim \frac{\bar{U}_{c}^{\prime}}{\bar{T}_{c}}\left\{\frac{\bar{\alpha}^{2}}{3} a^{ \pm} \phi_{a}+\phi_{b}+\frac{\bar{\alpha}^{2}}{3} \widehat{k} \ln |\eta| \phi_{a}\right\}
$$


where $\eta \equiv r-r_{c} \ll 1$ and

$$
\phi_{a}=\eta^{3}-\frac{3}{4} \chi_{a} \eta^{4}+\ldots, \quad \phi_{b}=1-\frac{1}{2} \bar{\alpha}^{2} \eta^{2}+\chi_{b} \eta^{4}+\ldots,
$$

with $\chi_{b}$ being given by (5.7) in $\mathrm{Wu}(2005)$, and

$$
\bar{\alpha}=\left(\alpha^{2}+m^{2} / r_{c}^{2}\right)^{1 / 2}, \quad \widehat{k}=\left(\frac{\bar{T}_{c}^{\prime}}{\bar{T}_{c}}-\frac{\bar{U}_{c}^{\prime \prime}}{\bar{U}_{c}^{\prime}}+\frac{1}{r_{c}}\right)-\frac{2 m^{2}}{\bar{\alpha}^{2} r_{c}^{3}}, \quad \chi_{a}=\frac{\bar{T}_{c}^{\prime}}{\bar{T}_{c}}-\frac{\bar{U}_{c}^{\prime \prime}}{\bar{U}_{c}^{\prime}}+\frac{1}{r_{c}} .
$$

Although our main focus will be on subsonic modes, for which $\widehat{k}=0$, the analysis will cover supersonic modes as well. The nonlinear evolution equation and the formulae for the low-frequency acoustic field apply equally to supersonic modes. Evidence that the same low-frequency radiation mechanism may operate in supersonic jets may be found in Hileman et al. (2005), where a strong correlation was found to exist between the far-field sound and the low-frequency density fluctuations.

For helical modes, the streamwise and spanwise velocity components both exhibit a singularity of a single-pole type at the critical level, i.e.

$$
u_{0} \sim\left(\frac{m^{2}}{\alpha^{2} r_{c}^{2}}\right) \frac{1}{\eta}, \quad w_{0} \sim-\left(\frac{\mathrm{i} m}{\alpha r_{c}}\right) \frac{1}{\eta} .
$$

Consideration of $\left(u_{1}, v_{1}, w_{1}, p_{1}, \theta_{1}\right)$ shows that $p_{1}$ satisfies an inhomogeneous Rayleigh equation, for which the solvability condition is derived as (Wu 2005)

$$
-\frac{r_{c}}{\bar{U}_{c}^{\prime}}\left\{3\left(c^{+}-c^{-}\right)-\left[\frac{2 \mathrm{i} \bar{\alpha}^{2}}{\alpha \bar{T}_{c}}\left(\frac{\partial A}{\partial \tilde{t}}+c \frac{\partial A}{\partial \tilde{x}}\right) \chi_{a}-\bar{\alpha}^{2} d\right]\left(a^{+}-a^{-}\right)\right\}=\frac{2 \mathrm{i}}{\alpha}\left[c I_{2} \frac{\partial A}{\partial \tilde{x}}+I_{3} \frac{\partial A}{\partial \tilde{t}}\right],
$$

where $I_{2}$ and $I_{3}$ are given in $\mathrm{Wu}(2005) ; d(\tilde{x}, \tilde{t})$ is an unknown function; and the jumps $\left(a_{+}-a_{-}\right)$and $\left(c_{+}-c_{-}\right)$have to be determined by analysing the critical-layer dynamics.

The singularity in the outer solution is to be smoothed out by reintroducing the nonequilibrium and viscous effects within the critical layer. As noted for example by $\mathrm{Wu}$ (2005), this thin region is locally flat because the radial variation is much more rapid than the azimuthal variation, and the dynamics is thus similar to (incompressible) planar shear layers considered in Wu et al. (1993). For the interaction of pairs of helical (oblique) modes, the critical layer is also minimally influenced by compressibility because although the temperature fluctuation acquires a large amplitude, it remains fairly passive in that its nonlinear effect on the amplitude evolution (Leib \& Lee $1995)$ is $O\left(R^{-1 / 3}\right)$ weaker than the dominant contribution by the velocity fluctuations - although in the case of a single helical/oblique or axisymmetric/planar mode, the temperature fluctuation would contribute the leading-order nonlinear effect (Goldstein \& Leib 1989; Leib 1991; Churilov \& Shukhman 1994). It therefore suffices to give an outline of the theory, highlighting only the key aspects that are relevant for the generation of sound.

The appropriate local transverse coordinate is

$$
Y=\left(r-r_{c}\right) / R^{-1 / 3},
$$


and the solution for the disturbance expands as

$$
\left.\begin{array}{rl}
u & =\epsilon R^{1 / 3} U_{1}(Y, \tilde{x}, \tilde{t}) E \cos m \phi+\epsilon U_{m}(Y, \tilde{x}, \tilde{t}) \cos m \phi+\ldots, \\
w & =\epsilon R^{1 / 3} W_{1}(Y, \tilde{x}, \tilde{t}) E \sin m \phi+\epsilon R^{-1 / 3} W_{m}(Y, \tilde{x}, \tilde{t}) \sin 2 m \phi+\ldots, \\
\theta & =\epsilon R^{1 / 3} \Theta_{1}(Y, \tilde{x}, \tilde{t}) E \cos m \phi+\epsilon \Theta_{m}(Y, \tilde{x}, \tilde{t}) \cos 2 m \phi+\ldots, \\
v & =\epsilon\left(\mathrm{i} \bar{\alpha}^{2} / \alpha\right) \eta A E \cos m \phi+\epsilon R^{-1 / 3} V_{m}(Y, \tilde{x}, \tilde{t}) \cos 2 m \phi+\ldots, \\
p & =\epsilon \frac{\bar{U}_{c}^{\prime}}{\bar{T}_{c}} A E \cos m \phi+\epsilon R^{-2 / 3}\left(p_{m}(\tilde{x}, \tilde{t})+R^{-1 / 3} P_{m}(Y, \tilde{x}, \tilde{t})\right) \cos 2 m \phi+\ldots,
\end{array}\right\}
$$

where we have put $\bar{\alpha}=\left(\alpha^{2}+m^{2} / r_{c}^{2}\right)^{1 / 2}$. The leading-order solution for $v$ and $p$ is just the trivial continuation of the outer expansion, but the streamwise and spanwise velocities as well as the temperature/density acquire a magnitude larger by a factor $R^{1 / 3}$ than that outside the critical layer. They are governed by equations

$$
\mathscr{L}\left(U_{1}, W_{1}, \Theta_{1}\right)=\left(\frac{\mathrm{i} m^{2} \bar{U}_{c}^{\prime}}{\alpha r_{c}^{2}}, \frac{m \bar{U}_{c}^{\prime}}{r_{c}}, \frac{\mathrm{i} \bar{\alpha}^{2} \bar{T}_{c}^{\prime}}{\alpha}\right) \tilde{A},
$$

where

$$
\mathscr{L}=\frac{\partial}{\partial \tilde{t}}+c \frac{\partial}{\partial \tilde{x}}+\mathrm{i} \bar{U}_{c} Y \frac{\partial}{\partial x}-\mu_{c} T_{c} \frac{\partial^{2}}{\partial Y^{2}} .
$$

The solution is (cf. Wu et al. 1993; Leib \& Lee 1995)

$$
\left(U_{1}, W_{1}, \Theta_{1}\right)=\left(\frac{\mathrm{i} m^{2} \bar{U}_{c}^{\prime}}{\alpha c r_{c}^{2}}, \frac{m \bar{U}_{c}^{\prime}}{c r_{c}}, \frac{\mathrm{i} \bar{\alpha}^{2} \bar{T}_{c}^{\prime}}{\alpha c}\right) \int_{0}^{\infty} \mathrm{e}^{-s \xi^{3}-\mathrm{i}\left(\alpha \bar{U}_{c}^{\prime} / c\right) Y \xi} \tilde{A}(\tilde{x}-\xi, \tilde{t}-\xi / c) \mathrm{d} \xi,
$$

where

$$
s=\frac{1}{3} \alpha^{2} \bar{U}_{c}^{\prime 2}\left(T_{c} \mu_{c} / c^{3}\right) .
$$

The mutual interaction between the helical pair induces an azimuthally dependent mean-flow distortion, $\left(U_{m}, V_{m}, W_{m}, \Theta_{m}\right)$, among which the axial velocity

$$
\begin{array}{r}
U_{m}=-\frac{\bar{\alpha}^{2} \bar{U}_{c}^{\prime 2}}{\alpha c^{3}}\left(\frac{m}{r_{c}}\right)^{2} \int_{0}^{\infty} \int_{0}^{\infty} \mathrm{e}^{-\mathrm{i} \bar{U}_{c}^{\prime} Y \xi /(2 c)} \frac{\sin \left[\bar{U}_{c}^{\prime} Y \xi /(2 c)\right]}{\xi} I_{u}(\xi, \eta ; s) \tilde{A}^{*}(\tilde{x}-\eta, \tilde{t}-\eta / c) \\
\quad \times \tilde{A}(\tilde{x}-\eta-\xi, \tilde{t}-\eta / c-\xi / c) \mathrm{d} \xi \mathrm{d} \eta+\text { c.c. }+\mathscr{D}_{m}(\tilde{x}, \tilde{t}),
\end{array}
$$

where $\mathscr{D}_{m}$ will have to be determined by matching with the global outer solution (see $\S 5.2$ ). It can be shown that as $Y \rightarrow \infty$ (cf. (3.49) in Wu et al. 1993),

$$
U_{m} \rightarrow \pm \frac{1}{2} \mathscr{J}_{u}(\tilde{x}, \tilde{t})+\mathscr{D}_{m},
$$

where we have put

$$
\mathscr{J}_{u}(\tilde{x}, \tilde{t})=-16 \pi \bar{U}_{c}^{\prime 2}\left(m / r_{c}\right)^{4} /\left(\alpha c^{3}\right) \int_{0}^{\infty} K_{u}(\eta)|\tilde{A}(\tilde{x}-\eta, \tilde{t}-\eta / c)|^{2} \mathrm{~d} \eta,
$$

with

$$
K_{u}(\eta)=\int_{0}^{\eta}(\eta-\zeta) \mathrm{e}^{-2 s \zeta^{3}} \mathrm{~d} \zeta
$$

Clearly, $\mathscr{J}_{u}(\tilde{x}, \tilde{t})$ represents a streamwise velocity jump, $\mathscr{J}_{u}(\tilde{x}, \tilde{t}) \equiv u_{m}\left(r_{c}^{+}, \tilde{x}, \tilde{t}\right)-$ $u_{m}\left(r_{c}^{-}, \tilde{x}, \tilde{t}\right)$, through which an $O(\epsilon)$ 'mean' streamwise velocity $u_{m}$ is generated in the main part of the jet (cf. Goldstein \& Choi 1989). A similar large 'mean temperature' is also induced (cf. Leib \& Lee 1995). 
The analysis of the critical layer (Wu 2005) shows that

$$
\begin{gathered}
a^{+}-a^{-}=-\widehat{k} \pi \mathrm{i}, \\
c^{+}-c^{-}=-\frac{\bar{\alpha}^{2}}{3 \bar{T}_{c}}\left[\frac{\mathrm{i}}{\alpha}\left(\frac{\partial \tilde{A}}{\partial \tilde{t}}+c \frac{\partial \tilde{A}}{\partial \tilde{x}}\right)\right] j \pi \mathrm{i}-\frac{\bar{\alpha}^{2}}{3} \widehat{k} d \pi \mathrm{i}+\mathscr{J}_{\mathcal{N}} .
\end{gathered}
$$

It may be noted that $\left(a^{+}-a^{-}\right)$and the linear part of $\left(c^{+}-c^{-}\right)$correspond to the familiar $(-\pi)$ phase jump in the logarithmic singularity in the outer solution for $p_{0}$ and $p_{1}$ respectively. The nonlinear part of $\left(c^{+}-c^{-}\right), \mathscr{J}_{\mathscr{N}}$, can be inferred from (3.84) in Wu et al. (1993), by identifying the spanwise wavenumber $\beta$ with $m / r_{c}$, or $\sin \theta=m /\left(\bar{\alpha} r_{c}\right)$. The resulting jump is inserted into (3.8) to obtain the amplitude equation

$$
\begin{aligned}
\frac{\partial \tilde{A}}{\partial \tilde{x}}+c_{g}^{-1} & \frac{\partial \tilde{A}}{\partial \tilde{t}}=\sigma \Delta \tilde{A}+l \int_{0}^{\infty} \int_{0}^{\infty} K(\xi, \eta ; s) \tilde{A}(\tilde{x}-\xi, \tilde{t}-\xi / c) \\
& \times \tilde{A}(\tilde{x}-\xi-\eta, \tilde{t}-\xi / c-\eta / c) \tilde{A}^{*}(\tilde{x}-2 \xi-\eta, \tilde{t}-2 \xi / c-\eta / c) \mathrm{d} \xi \mathrm{d} \eta,
\end{aligned}
$$

where the coefficients of the linear terms, $c_{g}$ and $\sigma$, are given by (5.31) and (5.32) in $\mathrm{Wu}$ (2005). The coefficient for the nonlinear term is

$$
l=-\mathrm{i} \alpha r_{c}\left[\frac{\pi \alpha \bar{U}_{c}^{\prime}\left|\bar{U}_{c}^{\prime}\right|^{3}}{\bar{T}_{c} c^{5}}\left(\alpha^{2}+\frac{m^{2}}{r_{c}^{2}}\right)^{2} \frac{m^{2}}{r_{c}^{2}}\right] /\left(c \bar{U}_{c}^{\prime} G\right),
$$

with $G$ being given on page 143 of Wu (2005). The kernel function $K$ is given by (3.85) of Wu et al. (1993).

Equation (3.18) is to be solved subject to an initial condition

$$
\tilde{A} \rightarrow \tilde{a}_{0} \mathrm{e}^{\sigma \Delta \tilde{x}} \tilde{A}_{l}\left(\tilde{x}-c_{g} \tilde{t}\right),
$$

where $\tilde{a}_{0}$ is the scaled amplitude of the disturbance at $\tilde{x}=0$, i.e. at $x_{3}=x_{n}+R^{-1 / 3} \Delta$. The parameters $\tilde{a}_{0}$ and $\Delta$ are not independent because $\Delta$ (which measures the scaled distance to the neutral position) has so far only been loosely defined. In order to specify the latter precisely, let $a_{0}$ denote the unscaled nominal amplitude exactly at the neutral position that the disturbance would have attained via its linear growth. Then $a_{0}$ and $\tilde{a}_{0}$ are related by the relation

$$
a_{0}=R^{-1} \tilde{a}_{0} \exp \left\{R \int_{x_{3}+R^{-1 / 3} \Delta}^{x_{3}} \alpha\left(\tilde{x}_{3}\right) \mathrm{d} \tilde{x}_{3}\right\}=R^{-1} \tilde{a}_{0} \exp \left\{-\frac{1}{2} \sigma R^{1 / 3} \Delta^{2}\right\},
$$

where $\alpha$ is approximated by its Taylor expansion about $x_{n}=0$. We can then take $\tilde{a}_{0}=1$ by choosing $\Delta$ to satisfy

$$
a_{0}=R^{-1} \exp \left\{-\frac{1}{2} \sigma R^{1 / 3} \Delta^{2}\right\},
$$

which gives a precise definition of $\Delta$. Strictly speaking, $a_{0}$ represents the 'saturation amplitude' projected on the basis of linear growth but will be referred to as 'initial amplitude' in view of (3.21).

Our principal interest is in the sound that may be radiated by such an instability wavepacket/wavetrain that undergoes amplification, followed by attenuation and decay. If the modes are supersonic, their eigenfunction is oscillatory and decays algebraically like $r^{-1 / 2}$ rather than exponentially, and they emit sound directly in the form of Mach waves (Tam \& Burton 1984; Tam 1995; Wu 2005). The aim of the present study is to demonstrate that an instability wavepacket modulated simultaneously in time and space radiates low-frequency sound waves whose time 
and length scales are comparable with those of the envelope, regardless of whether the modes are supersonic or subsonic.

\subsection{The nonlinearly generated slowly breathing mean field}

An important feature of the nonlinear interaction between a pair of helical modes is that it generates a large three-dimensional mean-flow distortion, with the magnitude of the streamwise velocity and the temperature and density components all being comparable with that of the fundamental modes (cf. Goldstein \& Choi 1989; Leib \& Lee 1995). Unlike the steady distortion in these paper, the nonlinearly induced mean field in the present study is slowly breathing in time due to the temporal modulation of the wavepacket. This unsteadiness is of course crucial for generation of sound. The mean field is governed by the equations

$$
\begin{aligned}
\bar{R}\left\{\frac{\partial u_{m}}{\partial \tilde{x}}+\right. & \left.\frac{\partial v_{m}}{\partial r}+\frac{v_{m}}{r}+\frac{2 m}{r} w_{m}\right\}+\frac{\partial \rho_{m}}{\partial \tilde{t}}+\bar{U} \frac{\partial \rho_{m}}{\partial \tilde{x}}=0 \\
& \left\{\frac{\partial u_{m}}{\partial \tilde{t}}+\bar{U} \frac{\partial u_{m}}{\partial \tilde{x}}\right\}+\bar{U}^{\prime} v_{m}=0 \\
& \bar{R}\left\{\frac{\partial v_{m}}{\partial \tilde{t}}+\bar{U} \frac{\partial v_{m}}{\partial \tilde{x}}\right\}=-\frac{\partial p_{m}}{\partial r} \\
& \bar{R}\left\{\frac{\partial w_{m}}{\partial \tilde{t}}+\bar{U} \frac{\partial w_{m}}{\partial \tilde{x}}\right\}=\frac{2 m}{r} p_{m} \\
& \left\{\frac{\partial \theta_{m}}{\partial \tilde{t}}+\bar{U} \frac{\partial \theta_{m}}{\partial \tilde{x}}\right\}+\bar{T}^{\prime} v_{m}=0
\end{aligned}
$$

supplemented by the state equation

$$
\bar{R} \theta_{m}+\bar{T} \rho_{m}=0
$$

These are homogeneous equations. The forcing comes from the critical layer through the velocity jump across the critical layer, which can easily be inferred from the planar case, i.e. (3.49) in (Wu et al. 1993), by replacing $\beta$ by $m / r_{c}$.

Eliminating $u_{m}, v_{m}, w_{m}, \theta_{m}$ and $\rho_{m}$ in favour of $p_{m}$, we obtain

$$
\left\{\frac{\partial}{\partial \tilde{t}}+\bar{U} \frac{\partial}{\partial \tilde{x}}\right\}\left\{\frac{\partial^{2}}{\partial r^{2}}+\frac{1}{r} \frac{\partial}{\partial r}-\frac{4 m^{2}}{r^{2}}+\frac{\bar{T}^{\prime}}{\bar{T}} \frac{\partial}{\partial r}\right\} p_{m}-2 \bar{U}^{\prime} \frac{\partial^{2} p_{m}}{\partial \tilde{x} \partial r}=0 .
$$

With the second-order derivative with respect to $\tilde{x}$ being absent, this is the longwavelength limit of the compressible Rayleigh equation. Across the critical layer, the pressure $p_{m}$ is continuous, but it follows from (3.15), (3.24) and (3.25) that the pressure gradient exhibits a jump

$$
p_{m}^{\prime}\left(r_{c}^{+}, \tilde{x}, \tilde{t}\right)-p_{m}^{\prime}\left(r_{c}^{-}, \tilde{x}, \tilde{t}\right)=-\frac{\bar{R}_{c}}{\bar{U}_{c}^{\prime}}\left(\frac{\partial}{\partial \tilde{t}}+c \frac{\partial}{\partial \tilde{x}}\right)^{2} \mathscr{J}_{u}(\tilde{x}, \tilde{t}) \equiv \mathscr{J}_{p}(\tilde{x}, \tilde{t}),
$$

where by integration by parts, we find that

$$
\mathscr{J}_{p}(\tilde{x}, \tilde{t})=j_{0} \int_{0}^{\infty} \mathrm{e}^{-2 s \eta^{3}}|\tilde{A}(\tilde{x}-\eta, \tilde{t}-\eta / c)|^{2} \mathrm{~d} \eta,
$$

with $j_{0}=16 \pi \bar{R}_{c} \bar{U}_{c}^{\prime}\left(m / r_{c}\right)^{4} /(\alpha c)$. The jump $\mathscr{J}_{p}$ acts as a radially compact, lowfrequency 'physical' source embedded in a shear flow. 
As $r \rightarrow 0, \bar{U}^{\prime}, \bar{T}^{\prime} \rightarrow 0$ so that (3.29) reduces simply to the Laplace equation

$$
\left\{\frac{\partial^{2}}{\partial r^{2}}+\frac{1}{r} \frac{\partial}{\partial r}-\frac{4 m^{2}}{r^{2}}\right\} p_{m}=0
$$

and the regularity requirement implies that

$$
p_{m} \sim \mathscr{C}_{0}(\tilde{x}, \tilde{t}) r^{2 m} \quad \text { as } \quad r \rightarrow 0 .
$$

The same limiting equation holds for $r \gg 1$, and it follows that

$$
p_{m} \sim \frac{\mathscr{B}(\tilde{x}, \tilde{t})}{r^{2 m}} \quad \text { as } \quad r \rightarrow \infty,
$$

where $\mathscr{C}_{0}(\tilde{x}, \tilde{t})$ and $\mathscr{B}(\tilde{x}, \tilde{t})$ are functions to be determined numerically. Obviously, helical modes with azimuthal wavenumbers $m \pm 1$ are most efficient in radiating sound.

As will become clear later, it is the Fourier transform of $\mathscr{B}(\tilde{x}, \tilde{t})$ that is needed for the purpose of calculating the radiated sound. Thus we take the Fourier transform of (3.29) and (3.30) with respect to both $\tilde{x}$ and $\tilde{t}$ to obtain

$$
\begin{gathered}
\left\{\frac{\partial^{2}}{\partial r^{2}}+\frac{1}{r} \frac{\partial}{\partial r}-\frac{4 m^{2}}{r^{2}}+\left(\frac{\bar{T}^{\prime}}{\bar{T}}-\frac{2 \bar{U}^{\prime}}{\bar{U}+\omega / k}\right) \frac{\partial}{\partial r}\right\} \widehat{p}_{m}(k, \omega)=0, \\
\widehat{p}_{m}^{\prime}\left(r_{c}^{+}, k, \omega\right)-\widehat{p}_{m}^{\prime}\left(r_{c}^{-}, k, \omega\right)=\widehat{\mathscr{J}}_{p}(k, \omega) .
\end{gathered}
$$

Equation (3.34) has two linearly independent solutions, $Y_{1}(r)$ and $Y_{2}(r)$, say. Without losing generality, we may require

$$
Y_{1} \sim r^{2 m} \text { for } r \ll 1 \text { and } Y_{2} \sim r^{-2 m} \text { for } r \gg 1,
$$

for convenience. Then the solution for $\widehat{p}$ can be expressed as a linear combination

$$
\widehat{p}_{m}=C_{1}^{ \pm} Y_{1}(r)+C_{2}^{ \pm} Y_{2}(r)
$$

where \pm indicates that the constants $C_{1}^{ \pm}$and $C_{2}^{ \pm}$take different values depending on $r>r_{c}$ or $r<r_{c}$. Since $Y_{1} \sim r^{2 m}$ for $r \gg 1$ and $Y_{2} \sim r^{-2 m}$ for $r \ll 1$, one has to set $C_{1}^{+}=0$ and $C_{2}^{-}=0$ to render $\widehat{p}_{m}$ bounded at infinity and $r=0$. Now applying the continuity of $\widehat{p}_{m}$ and the jump condition, we have

$$
C_{2}^{+} Y_{2}\left(r_{c}\right)=C_{1}^{-} Y_{1}\left(r_{c}\right), \quad C_{2}^{+} Y_{2}^{\prime}\left(r_{c}\right)-C_{1}^{-} Y_{1}^{\prime}\left(r_{c}\right)=\widehat{\mathscr{J}}_{p}(k, \omega),
$$

from which it follows

$$
\widehat{\mathscr{B}}(k, \omega)=C_{2}^{+}=\widehat{\mathscr{J}}_{p}(k, \omega) Y_{1}\left(r_{c}\right) /\left(Y_{1} Y_{2}^{\prime}-Y_{2} Y_{1}^{\prime}\right) .
$$

Functions $Y_{1}$ and $Y_{2}$ have to be found by numerically solving (3.34), which is essentially the long-wavelength limit of the compressible Rayleigh equation. The equation is singular at the radial position $\hat{r}_{c}$ where $\bar{U}\left(\hat{r}_{c}\right)=-\omega / k$; here $\hat{r}_{c}$ may be referred to as the 'envelope critical layer' in order to distinguish it from the usual (phase) critical layer $r_{c}$. A critical layer of the former type appears in certain generalized formulation of acoustic analogy (Goldstein \& Leib 2008), where the singularity has to be removed by reintroducing the weak non-parallel effect of the base flow, since all other terms are predesignated as sources. In our study, the signature $p_{m}$ is of hydrodynamic nature for $r=O(1)$, and thus viscous effects are at our disposal and can be used to smooth out the singularity. It turns out that for evaluating the noise radiated from a subsonic jet, one only needs $Y_{1}$ and $Y_{2}$ for those values of $k$ 
and $\omega$ for which an envelope critical layer does not arise. In this case, $Y_{1}$ and $Y_{2}$ can be obtained by integrating (3.34) in a straightforward manner using the respective asymptotes in (3.36) for small and large $r$ as boundary conditions.

\subsection{The acoustic far field}

The long-wavelength Rayleigh equation (3.29) governing the slowly modulating mean flow is no longer valid in the far field corresponding to $r=O\left(R^{1 / 3}\right)$, because the transverse and streamwise length scales become comparable. We thus introduce the radial variable

$$
\tilde{r}=R^{-1 / 3} r \text {. }
$$

For $\tilde{r}=O(1)$, the instability modes and their harmonics all have completely diminished owing to exponential transverse attenuation. In contrast, the slowly breathing mean field, which decays algebraically, acquires the character of sound, and the solution expands as

$$
(u, v, w, p, \theta, \rho)=\epsilon R^{-4 / 3}\left(\tilde{u}_{s}, \tilde{v}_{s}, \tilde{w}_{s}, \tilde{p}_{s}, \tilde{\theta}_{s}, \tilde{\rho}_{s}\right)+\ldots
$$

As expected, functions $\tilde{u}_{s}, \tilde{v}_{s}$, etc. satisfy the standard linearized equations for an acoustic perturbation in a uniform background flow $\bar{U}=0$ and $\bar{T}=T_{a}$. Specifically, the governing equation for $\tilde{p}_{s}$ is

$$
M_{a}^{2} \frac{\partial^{2} \tilde{p}_{s}}{\partial \tilde{t}^{2}}-\left\{\frac{\partial^{2}}{\partial \tilde{r}^{2}}+\frac{1}{\tilde{r}} \frac{\partial}{\partial \tilde{r}}-\frac{4 m^{2}}{\tilde{r}^{2}}+\frac{\partial^{2}}{\partial \tilde{x}^{2}}\right\} \tilde{p}_{s}=0,
$$

where the acoustic Mach number $M_{a}=M / T_{a}^{1 / 2}$ with $T_{a}$ being the ambient air temperature. For $\tilde{r} \ll 1, \tilde{p}_{m} \sim \mathscr{B}(\tilde{x}, \tilde{t}) / \tilde{r}^{2 m}$ in order to match with (3.33). This indicates that $\mathscr{B}(\tilde{x}, \tilde{t})$ acts as the 'apparent' acoustic source, which can be expressed via (3.39) in terms of the physical source $\mathscr{J}_{p}$. The source is non-compact in the axial direction because its spatial extent is comparable with the wavelength of the emitted sound, both being $O\left(R^{1 / 3} R_{J}\right)$.

On taking the Fourier transform with respect to both $\tilde{x}$ and $\tilde{t},(3.41)$ reduces to the Helmholtz equation

$$
\left\{\frac{\partial^{2}}{\partial \tilde{r}^{2}}+\frac{1}{\tilde{r}} \frac{\partial}{\partial \tilde{r}}+\left(M_{a}^{2} \omega^{2}-k^{2}-\frac{4 m^{2}}{\tilde{r}^{2}}\right)\right\} \widehat{p}_{s}=0,
$$

subject to the matching condition

$$
\widehat{p}_{s} \rightarrow \frac{\widehat{\mathscr{B}}(k, \omega)}{\tilde{r}^{2 m}} \quad \text { as } \quad \tilde{r} \rightarrow 0,
$$

with $\widehat{\mathscr{B}}(k, \omega)$ denoting the Fourier transform of $\mathscr{B}(\tilde{x}, \tilde{t})$.

The appropriate solution to (3.42) and (3.43) can be expressed as

$$
\widehat{p}_{s}=q(k, \omega) H_{2 m}^{(1)}(\mathscr{K} \tilde{r}),
$$

where $H_{2 m}^{(1)}$ denotes the first-kind Hankel function of order $2 m$ and

$$
\mathscr{K}(k, \omega)=\left(M_{a}^{2} \omega^{2}-k^{2}\right)^{1 / 2} .
$$

By matching, the coefficient $q(k, \omega)$ is found to be

$$
q(k, \omega)=\frac{2^{2 m} \pi}{(2 m) !} \mathscr{K}^{2 m}(k, \omega) \widehat{\mathscr{B}}(k, \omega) .
$$


The inversion of the Fourier transform then yields the acoustic pressure in physical space,

$$
\tilde{p}_{s}=\frac{2^{2 m} \pi}{(2 m) !} \int_{-\infty}^{\infty} \int_{-\infty}^{\infty} \mathscr{K}^{2 m}(k, \omega) \widehat{\mathscr{B}}(k, \omega) H_{2 m}^{(1)}(\mathscr{K} \tilde{r}) \mathrm{e}^{-\mathrm{i}(k \tilde{x}+\omega \tilde{t})} \mathrm{d} k \mathrm{~d} \omega .
$$

For $\tilde{r} \gg 1$, we may approximate $H_{2 m}^{(1)}$ by its asymptotic expansion to obtain

$$
\begin{aligned}
\tilde{p}_{s} \sim & \frac{2^{2 m} \pi}{(2 m) !}\left(\frac{2}{\pi \tilde{r}}\right)^{1 / 2} \mathrm{e}^{-\mathrm{i}(m \pi+\pi / 4)} \\
& \times \int_{-\infty}^{\infty} \int_{-\infty}^{\infty} \mathscr{K}^{2 m-1 / 2}(k, \omega) \widehat{\mathscr{B}}(k, \omega) \exp \{-\mathrm{i}(k \tilde{x}-\mathscr{K} \tilde{r})-\mathrm{i} \omega \tilde{t}\} \mathrm{d} k \mathrm{~d} \omega .
\end{aligned}
$$

Of primary interest is the far field of the acoustic region, corresponding to

$$
\tilde{R}=\left(\tilde{r}^{2}+\tilde{x}^{2}\right)^{1 / 2} \gg 1 .
$$

The acoustic pressure there can be approximated by using the stationary-phase method. The phase of the integrand, $\phi(k) \equiv k \cos \theta-\mathscr{K}(k, \omega) \sin \theta$, has a stationary point at

$$
k=k_{s}=-M_{a} \omega \cos \theta,
$$

where $\theta=\tan ^{-1}(\tilde{r} / \tilde{x})$. Thus the instantaneous pressure in the far field is given by

$$
\tilde{p}_{s} \sim \frac{2^{2 m+1} \pi}{(2 m) ! \tilde{R}} \mathrm{e}^{-\mathrm{i}(m \pi+\pi / 2)}(\sin \theta)^{2 m} \int_{-\infty}^{\infty}\left(M_{a} \omega\right)^{2 m} \widehat{\mathscr{B}}\left(-M_{a} \omega \cos \theta, \omega\right) \mathrm{e}^{\mathrm{i} \omega(M \tilde{R}-\tilde{t})} \mathrm{d} \omega .
$$

To obtain $\widehat{\mathscr{B}}\left(-M_{a} \omega \cos \theta, \omega\right)$, one has to solve (3.34). Since the equation depends on $\omega / k_{s}$, which is a function of $\theta$ but independent of $\omega$, we may write

$$
\widehat{\mathscr{B}}\left(-M_{a} \omega \cos \theta, \omega\right)=\mathscr{T}(\theta) \widehat{\mathscr{J}}_{p}\left(-M_{a} \omega \cos \theta, \omega\right),
$$

where $\mathscr{T}(\theta)$ is determined by solving (3.34) with $-\omega / k=1 /\left(M_{a} \cos \theta\right)$, subjected to a unit jump, namely

$$
\left.\begin{array}{r}
\left\{\frac{\partial^{2}}{\partial r^{2}}+\frac{1}{r} \frac{\partial}{\partial r}-\frac{4 m^{2}}{r^{2}}+\left(\frac{\bar{T}^{\prime}}{\bar{T}}-\frac{2 \bar{U}^{\prime}}{\bar{U}-1 /\left(M_{a} \cos \theta\right)}\right) \frac{\partial}{\partial r}\right\} \widehat{p}_{m}=0, \\
\widehat{p}_{m}\left(r_{c}^{+}\right)-\widehat{p}_{m}\left(r_{c}^{-}\right)=0, \quad \widehat{p}_{m}^{\prime}\left(r_{c}^{+}\right)-\widehat{p}_{m}^{\prime}\left(r_{c}^{-}\right)=1, \\
\widehat{p} \sim r^{2 m} \quad \text { as } \quad \tilde{r} \rightarrow 0, \quad \widehat{p} \rightarrow \frac{\mathscr{T}(\theta)}{r^{2 m}} \quad \text { as } \quad r \rightarrow \infty .
\end{array}\right\}
$$

There is no need to consider an envelope critical layer in a subsonic jet since $\left|\omega / k_{s}\right|=\left|1 /\left(M_{a} \cos \theta\right)\right|>1 \geqslant \bar{U}$. As $\mathscr{T}(\theta)$ relates the forcing $\widehat{\mathscr{J}}_{p}$ to the output $\widehat{\mathscr{B}}$, it will be referred to as a 'transfer function'. It is determined by the velocity and temperature profiles of the basic flow and the critical level $r_{c}$, which in turn depends on the 'carrier-wave frequency' $\omega_{c}$. Clearly, $\mathscr{T}(\theta)$ characterizes the basic-flow refraction (shielding and amplification) effects (cf. Goldstein 1975), which are present despite the fact that the characteristic wavelength is much larger than the width of the shear layer.

The radiated sound is of broadband nature. It follows from (3.50) and (3.51) that its normalized spectrum at an arbitrary point $(\tilde{R}, \theta)$ (polar coordinates) can be defined as

$$
\mathscr{I}(\omega ; \theta)=\omega^{4 m}\left|\widehat{\mathscr{J}}_{p}\left(-M_{a} \omega \cos \theta, \omega\right)\right|^{2} .
$$


The intensity of the acoustic pressure at an arbitrary point $(\tilde{R}, \theta)$ is then given by the root-mean-square value

$$
\sqrt{\overline{\tilde{p}_{s}^{2}}}=\frac{2^{2 m+1} \pi}{(2 m) !} M_{a}^{2 m} \frac{\mathscr{D}(\theta)}{\tilde{R}},
$$

where the directivity function $\mathscr{D}(\theta)$ is given by

$$
\mathscr{D}(\theta)=\mathscr{T}(\theta)(\sin \theta)^{2 m}\left[\int_{-\infty}^{\infty} \omega^{4 m}\left|\widehat{\mathscr{F}}_{p}\left(-M_{a} \omega \cos \theta, \omega\right)\right|^{2} \mathrm{~d} \omega\right]^{1 / 2} .
$$

Note that (3.41) remains invariant if $\tilde{x}, \tilde{r}$ and $\tilde{t}$ are rescaled by the same factor. This means that while the results for the acoustic field are expressed in terms of the Fourier transforms with respect to $\tilde{x}$ and $\tilde{t}$, they would remain valid if the Fourier transforms with respect to some simultaneously renormalized variables, e.g. $\bar{x}$ and $\bar{t}$ (see $\S 4$ and $\S 5.4$ ), are substituted in, with the only difference being some multiplicative factors. This property will be used later.

In general, a numerical procedure is required to evaluate the directivity and spectrum of the acoustic field radiated by a wavepacket. The analysis can be taken a step further for a linear wavepacket with a Gaussian spectrum, as will be shown in $\S 5.4$.

\subsection{An appraisal of the mechanism}

It is worth noting that the mathematical problem of the acoustic radiation is somewhat similar to that of a multipolar source embedded in a shear flow (Balsa 1975; Goldstein $1975,1976)$, in that the sources are radially compact and of low frequency. However, the crucial differences are $(a)$ that the physical source $\mathscr{J}_{p}$ in the present problem is unambiguously identified by analysing the hydrodynamic field and $(b)$ that the source is non-compact in the axial direction and thus cannot be modelled appropriately by acoustic multipoles.

Although the wavepacket is modulated over a length scale much longer than its wavelength as in the theories of Tam \& Morris (1980) and Crighton \& Huerre (1990), the present mechanism is fundamentally different from theirs. Firstly, the intensity of the radiated sound waves is algebraically rather than exponentially small. Secondly, the characteristic frequency of the sound emitted is comparable with the beating frequencies, rather than the main frequency, of the instability modes. Thirdly, the generation of sound waves involves an 'inverse energy cascade': the hydrodynamic energy in relatively short-scale wave motions is first transferred to the unsteady mean-flow distortion of large scale before being radiated. Since sound waves are emitted through the streaming effect and the source is a functional of the envelope, the mechanism may be referred to as 'streaming acoustics' or 'envelope radiation'. The latter is suggested here also because the general wave mechanics involved is quite similar to the envelope-radiation mechanism proposed by Fritt (1982) and Chimonas \& Grant (1984) for the generation of gravity waves, where two Kelvin-Helmholtz waves with short but similar wavelengths interact to generate a difference-wavenumber mode of long wavelength, which radiates as a gravity wave (Scinocca \& Ford 2000). An interaction of this kind between two two-dimensional temporally evolving instability modes on a subsonic planar jet was recently explored in the framework of the acoustic analogy by Sandham, Morfey \& $\mathrm{Hu}$ (2006) and was found to be able to explain certain features of sound generation. In a more recent paper, Sandham \& Salgado (2008) further investigated the role of difference-frequency modes arising from interactions 
between spatially developing axisymmetric and helical modes on a circular jet (see further discussion below).

As a matter of fact, the interpretation of the upper-scale or inverse energy transfer in terms of the interaction of two purely sinusoidal eigenmodes is too simplistic because the Reynolds stresses produced often do not consist of significant supersonic components (see e.g. figure $6 b$ of Sandham \& Salgado 2008). It follows that the resulting beating mode, with a difference wavenumber or frequency, is not a radiating component and thus acoustically behaves more or less like the original eigenmodes (see further discussion below). In order to produce a radiating response, the interacting modes must be strongly modulated in both time and space over the beating time and length scales. Their interaction generates an unsteady slowly breathing mean field, and the inverse cascade is achieved via a streaming process. It should be pointed out that the inverse cascade is a hydrodynamic process in general. In our problem, it takes place within the critical layer (Wu et al. 1993) and so is influenced by both viscous and non-equilibrium effects at leading order. These effects are reflected respectively by the exponential kernel and the convolution in the resulting physical source, the pressure gradient jump $\mathscr{J}_{p}$ (see (3.30) and (3.31)). Since the physical source is embedded within the shear layer, the generation of sound is further influenced by the mean-flow refraction, the effect of which is properly characterized by a transfer function $\mathscr{T}$ (3.51).

It is interesting to contrast the present asymptotic approach with the acoustic analogy. When the latter is applied to the present problem, one may evaluate the so-called nonlinear source, i.e. the Reynolds stresses contributed by the wave-wave interaction, using the available solution for the instability mode and then solve (i) Lighthill's wave equation or (ii) Lilley's equation, to predict the acoustic field. Either version of the acoustic analogy involves an inverse cascade of energy from the relatively high-frequency instability modes to the lower-frequency sound waves, but the fact that the calculation is based on a wave or wave-like equation implies that the inverse cascade is treated as an acoustic as opposed to a full hydrodynamic process. Obviously, method (i) ignores both the viscous and mean-flow refraction effects, while method (ii) includes the latter but neglects viscosity. Consequently, even if our analytical solution for the instability waves is used to compute the 'nonlinear source', the acoustic field predicted by method (i) or (ii) would differ at least quantitatively from the first-principles solution given in the present paper. Another example in which viscous effects play a leading-order role in the upper-scale energy transfer leading to sound radiation was described by $\mathrm{Wu} \& \mathrm{Hogg}$ (2006). These observations suggest that conventional descriptions of the inverse cascade by 'nonlinear forcing' acting on Lighthill's wave equation, or on Lilley's equation, may not always be adequate.

Since both the present work and that of Sandham \& Salgado (2008) address sound radiation from relatively low-frequency components driven by nonlinear wave-wave interactions, it is appropriate to comment on the relation between them. In the paper by Sandham \& Salgado (2008), Lilley's equation was employed to compute far-field sound waves radiated from the interactions between two axisymmetric modes, one axisymmetric mode and one helical mode and between two helical modes both with $m=1$. In each case, the forcing (or 'source') was taken to be the so-called streamwise quadrupole, which was further assumed to be located at the inflection point. None of these approximations can be justified in the context of acoustic analogy approach; it is not clear a priori that the combinations of modes chosen are most relevant or why only the steamwise quadrupole was retained and why it concentrates at the inflection point. In contrast, the present asymptotic approach, which is based on a 
detailed analysis of the hydrodynamic field and its radiating property, shows that the interaction between the helical pair $(m= \pm 1)$ is most efficient in the sense that it leads to an exceptionally large radiating 'mean distortion'; the forcing (or physical source) comes from the critical layer locally because dominant interactions take place there. Moreover, the contribution to the dominant streaming is not from the 'streamwise quadrupole' but from the 'spanwise quadrupole'. This can be deduced by noting that the jump (3.14) in this paper is essentially (3.49) of Wu et al. (1993), and the latter comes from $\hat{V}_{2, Y}^{(0,2)} \sim \hat{W}^{(0,2)}$ in $(3.45)$, which is driven by the 'spanwise quadrupole' (see (3.32)), while the 'streamwise quadrupole' $S_{11}^{(0,2)}$ in (3.45) does not contribute to jump (3.49).

Sandham \& Salgado (2008) found that for the combinations considered the streamwise quadrupole turned out to be strongest for the difference mode with $S t \approx 0.2$, apparently in agreement with the acoustic spectral peak observed in experiments. Unfortunately, the significance of this result is undermined by two facts: $(a)$ the spanwise quadrupole, which may be just as and even more important (see the discussion above), was neglected, and $(b)$ the streamwise quadrupole, which is modulated in space only, consists principally of subsonic components (see figure $6 b$ of Sandham \& Salgado). As the authors realized, the radiation efficiency is actually determined by small-amplitude supersonic components in its spectral tail (rather than by the overall strength of the quadrupole). In order to elaborate this point a little further, suppose that the instability modes have frequencies $\omega_{1}$ and $\omega_{2}\left(\omega_{1}>\omega_{2}\right)$ and streamwise wavenumbers $\alpha_{1}$ and $\alpha_{2}$. Then the 'nonlinear source' and hence the difference-frequency mode can be represented in the form

$$
D(X) \phi_{d}(r) \mathrm{e}^{\mathrm{i}\left(\alpha_{1}-\alpha_{2}\right) x-\mathrm{i}\left(\omega_{1}-\omega_{2}\right) t}+\text { c.c. },
$$

where the dependence on the azimuthal coordinate is suppressed. The functions $\phi_{d}(r)$ and $D(X)$ characterize respectively the radial variation and axial modulation over a slow variable $X \equiv \tilde{\epsilon} x$, where $\tilde{\epsilon} \ll 1$. Since the carrier wave has a subsonic phase speed $\left(\omega_{1}-\omega_{2}\right) /\left(\alpha_{1}-\alpha_{2}\right)<1 / M_{a}$, radiating supersonic components may arise only when the spatial modulation $D(X)$ is considered. Let $\widehat{D}(k)$ be the Fourier transform of $D(X)$ with respect to $X$. Typically, $\widehat{D}(k)$ is of order one for $k=O(1)$, but $\widehat{D}(k) \ll 1$ for large $k \gg O(1)$. The Fourier transform of (3.56) can be written as $\widehat{D}\left(k-\left(\alpha_{1}-\alpha_{2}\right) / \tilde{\epsilon}\right)$. A component with wavenumber $k$ is radiating if its phase speed $\left(\omega_{1}-\omega_{2}\right) /(\tilde{\epsilon} k)$ is supersonic, i.e. if $\left|\left(\omega_{1}-\omega_{2}\right) /(\tilde{\epsilon} k)\right|>1 / M_{a}$; the corresponding amplitude is $\widehat{D}\left(k_{s}\right)$, with $k_{s}=k-\left(\alpha_{1}-\alpha_{2}\right) / \tilde{\epsilon}$ in the interval

$$
-\left[M_{a}\left(\omega_{1}-\omega_{2}\right)+\left(\alpha_{1}-\alpha_{2}\right)\right] / \tilde{\epsilon}<k_{s}<\left[M_{a}\left(\omega_{1}-\omega_{2}\right)-\left(\alpha_{1}-\alpha_{2}\right)\right] / \tilde{\epsilon}<0 .
$$

Obviously, $\widehat{D}\left(k_{s}\right)$ must be very small, since $k_{s}=O(1 / \tilde{\epsilon}) \gg 1$ is in the spectral tail of $D$. The result shows that although the difference-frequency mode (3.56) may emit somewhat more efficiently than the original instability waves, the radiation might be viewed as being essentially due to the mechanism described by Crighton \& Huerre (1990) for a subsonically propagating spatially modulated wavetrain, which (3.56) is. In contrast, the slowly breathing mean distortion resulting from our streaming mechanism is modulated simultaneously in both time $\bar{t}$ and space $\bar{x}$ and decays algebraically in the radial direction (3.33). When it is Fourier transformed with respect to the slow variables $\bar{t}$ and $\bar{x}$, dominant components concentrate in the frequency-wavenumber domain $(\omega, k)$ in which $\omega=O(1)$ and $k=O(1)$. The radiating supersonic components $(\omega, k)=\left(\omega,-M_{a} \omega \cos \theta\right)$ (see (3.49)) are clearly within in this prime energy-containing domain. These components occupy two fan-shaped 
sub-regions defined by $|k|<M_{a}|\omega|$, implying that for $M_{a}=O(1)$ the slowly breathing mean distortion consists primarily of supersonic components.

\section{Non-parallelism and the composite amplitude equation}

\subsection{Non-parallel-flow regime}

In the non-equilibrium regime considered in $\S 3$, the nonlinear evolution of the instability modes takes place on a relatively short scale, for which non-parallelism is negligible at leading order. If the initial amplitude is reduced, nonlinear evolution would occur in an $O\left(R^{-1 / 2}\right)$ vicinity of the neutral position $x_{n}$, i.e.

$$
x_{3}=x_{n}+R^{-1 / 2} \bar{x} \quad \text { with } \quad \bar{x}=O(1) .
$$

Non-parallelism then becomes a leading-order effect in the sense that the linear growth rate of the modes varies over a length scale comparable with the length scale over which the amplitude evolves. In the region specified by (4.1), the local basic velocity and temperature profiles can be approximated, to the required order, by

$$
\left(\bar{U}\left(r, x_{3}\right), \bar{T}\left(r, x_{3}\right)\right) \approx\left(\bar{U}\left(r, x_{n}\right), \bar{T}\left(r, x_{n}\right)\right)+R^{-1 / 2}\left(\bar{U}_{1}(r), \bar{T}_{1}(r)\right) \bar{x} .
$$

Corresponding to the spatial variable $\bar{x}$ (see (4.1)), the slow-time variable to describe the simultaneous temporal modulation is

$$
\bar{t}=R^{-1 / 2} t .
$$

The nonlinear evolution in this regime is considered in $\mathrm{Wu}$ (2005), where it is shown that the threshold magnitude is

$$
\epsilon=O\left(R^{-7 / 6}\right)
$$

The disturbance may be expressed at leading order as

$$
(u, v, w, p, \theta, \rho)=\epsilon \bar{A}(\bar{x}, \bar{t})\left(p_{0}, u_{0}, v_{0}, w_{0}, \theta_{0}, \rho_{0}\right) E \cos m \phi+\text { c.c. }
$$

The evolution equation for $\bar{A}$, which is related to $\tilde{A}$ by $\bar{A}=R^{1 / 6} \tilde{A}$, is found to be

$$
\frac{\partial \bar{A}}{\partial \bar{x}}+c_{g}^{-1} \frac{\partial \bar{A}}{\partial \bar{t}}=\sigma \bar{x} \bar{A}+\bar{l} \bar{A} \int_{0}^{\infty}|\bar{A}(\bar{x}-\xi, \bar{t}-\xi / c)|^{2} \mathrm{~d} \xi,
$$

where

$$
\bar{l}=-\mathrm{i} \pi r_{c} /\left(c^{2} \bar{T}_{c} G\right) \alpha^{-2 / 3}\left|\bar{U}_{c}^{\prime}\right|^{1 / 3}\left(\mu_{c} T_{c}\right)^{-4 / 3}\left(\frac{2}{3}\right)^{2 / 3} \Gamma\left(\frac{1}{3}\right)\left(\alpha^{2}-\frac{m^{2}}{r_{c}^{2}}\right) \frac{m^{4}}{r_{c}^{4}} .
$$

The modulating mean flow in the main part of the jet remains unaltered, except that the jump (3.30) simplifies to

$$
\mathscr{J}_{p}=8 \pi\left(\frac{2}{3}\right)^{2 / 3} \Gamma\left(\frac{1}{3}\right) \bar{R}_{c} \alpha^{-5 / 3}\left|\bar{U}_{c}^{\prime}\right|^{1 / 3} \operatorname{sgn}\left(\bar{U}_{c}^{\prime}\right)\left(m / r_{c}\right)^{4}\left(T_{c} \mu_{c}\right)^{-1 / 3}|\bar{A}(\bar{x}, \bar{t})|^{2} .
$$

\subsection{The composite amplitude equation}

Equations (3.18) and (4.3) describe the nonlinear evolution in two distinguished regimes respectively: the former includes the non-equilibrium effect but neglects nonparallelism, while the latter does the opposite. It is desirable to construct a single composite amplitude equation, which includes both effects, thereby unifying the two 
regimes. This can be achieved by observing that the operator governing the criticallayer dynamics is

$$
R^{-1 / 6}\left(\frac{\partial}{\partial \bar{t}}+\frac{\partial}{\partial \bar{x}}\right)+\mathrm{i} \alpha \bar{U}_{c}^{\prime} Y-\mu_{c} T_{c} \frac{\partial^{2}}{\partial Y^{2}} .
$$

In the derivation of $(4.3)$, the $O\left(R^{-1 / 6}\right)$ non-equilibrium term was neglected, but it had to be reintroduced in a diffusion layer governing the mean-flow distortion (Wu et al. 1993). However, if this formally small term is retained within the critical layer, then we arrive at the amplitude equation

$$
\begin{aligned}
\frac{\partial \bar{A}}{\partial \bar{x}}+c_{g}^{-1} & \frac{\partial \bar{A}}{\partial \bar{t}}=\sigma \bar{x} \bar{A}+l R^{2 / 3} \int_{0}^{\infty} \int_{0}^{\infty} K(\xi, \eta ; \bar{s}) \bar{A}(\bar{x}-\xi, \bar{t}-\xi / c) \\
& \times \bar{A}(\bar{x}-\xi-\eta, \bar{t}-\xi / c-\eta / c) \bar{A}^{*}(\bar{x}-2 \xi-\eta, \bar{t}-2 \xi / c-\eta / c) \mathrm{d} \xi \mathrm{d} \eta,
\end{aligned}
$$

where $l$ is the same as (3.19) and $K$ is given by (3.85) of $\mathrm{Wu}$ et al. (1993) but with $\bar{s}=s R^{1 / 2}$. We shall assume that the disturbance upstream is of sufficiently small amplitude such that nonlinearity is negligible. The appropriate initial condition then is of the form

$$
\bar{A} \rightarrow \bar{a}_{0} \mathrm{e}^{\left(\sigma \bar{x}^{-2}\right) / 2} \bar{A}_{l}\left(\bar{x}-c_{g} \bar{t}\right) \quad \text { as } \quad \bar{x} \rightarrow-\infty,
$$

where $\bar{A}_{l}=O(1)$ and $\bar{a}_{0}$ is the rescaled overall amplitude of the disturbance.

Alternatively, a composite amplitude equation can be obtained in the nonequilibrium regime by retaining the $O\left(R^{-2 / 3}\right)$ term in (3.2), which was ignored in deriving (3.18). The linear term in (3.18) is then modified to $\sigma\left(\Delta+R^{-1 / 3} \tilde{x}\right) \tilde{A}$. The resulting equation, when written in terms of $\bar{x}$ by using the relation (see (3.1) and (4.1))

$$
\bar{x}=-R^{1 / 6} \Delta+R^{-1 / 6} \tilde{x},
$$

acquires the same form as (4.5). In the appendix, it is demonstrated that the evolution problem ((4.5) and (4.6)) accommodates both regimes, each of which is realized for a characteristic size of $\bar{a}_{0}$.

It is informative, as well as convenient for computational purpose, to introduce normalized variables

$$
\hat{x}=\left|\sigma_{r}\right|^{1 / 2} x, \quad \hat{t}=\left|\sigma_{r}\right|^{1 / 2} t, \quad A=R^{-7 / 6} \bar{A},
$$

in terms of which, the amplitude equation (4.5) and initial condition (4.6) are rewritten as

$$
\begin{aligned}
\frac{\partial A}{\partial \hat{x}}+c_{g}^{-1} \frac{\partial A}{\partial \hat{t}} & =\hat{\sigma} \hat{x} A+\hat{l} \int_{0}^{\infty} \int_{0}^{\infty} K(\xi, \eta ; \hat{s}) A(\hat{x}-\xi, \hat{t}-\xi / c) \\
\times A(\hat{x}-\xi-\eta, \hat{t}-\xi / c-\eta / c) A^{*}(\hat{x}-2 \xi-\eta, \hat{t}-2 \xi / c-\eta / c) \mathrm{d} \xi \mathrm{d} \eta & \\
A & \rightarrow a_{0} A_{l}\left(\hat{x}-c_{g} \hat{t}\right) \quad \text { as } \quad \hat{x} \rightarrow-\infty
\end{aligned}
$$

where

$$
\hat{\sigma}=\sigma /\left|\sigma_{r}\right|, \quad \hat{l}=R^{3} l /\left|\sigma_{r}\right|^{3}, \quad \hat{s}=s R^{1 / 2} /\left|\sigma_{r}\right|^{3 / 2} .
$$

Note that $A=R^{-1} \tilde{A}=R^{-7 / 6} \bar{A}$ is the unscaled amplitude, and the 'initial condition' $a_{0}$ is measured by the projected 'linear saturation amplitude' at the neutral position (see (3.21)). 


\section{Results and discussions}

In the numerical computation to be presented, the basic velocity profile is taken to be

$$
\bar{U}= \begin{cases}1, & r \leqslant h, \\ \exp \left\{-\left(\frac{r-h}{b}\right)^{2}\right\}, & r>h,\end{cases}
$$

which was suggested by Tam \& Burton (1984) and has been used in a number of papers including $\mathrm{Wu}$ (2005). Its applicability is restricted to the region before the end of the potential core, after which the axial velocity decreases with the downstream distance. For simplicity the Prandtl number is assumed to be unity so that the temperature profile is given by Crocco's relation

$$
\bar{T}=\left(1+\frac{\gamma-1}{2} M^{2}\right)\left[T_{a}+\left(1-T_{a}\right) \bar{U}\right]-\frac{\gamma-1}{2} M^{2} \bar{U}^{2} .
$$

The parameters $h$ and $b$ in (5.1) characterize the local centre and width of the shear layer respectively and are related by (6.3) in $\mathrm{Wu}(2005)$ in order to conserve the momentum. Both $h$ and $b$ are functions of $x_{3}$, but a global relation is not needed here, since the present analysis focuses on the vicinity of the neutral position $x_{n}$. The local variation is controlled by $\sigma_{s} \equiv b^{\prime}\left(x_{n}\right)$, which is related to the usual spreading rate of the 'half-velocity width' $b_{1 / 2}^{\prime}\left(x_{n}\right)$ by the relation

$$
\sigma_{s}=b_{1 / 2}^{\prime}\left(x_{n}\right) /\left(h^{\prime}(b)+\ln \sqrt{2}\right) .
$$

\subsection{Characteristics of subsonic instability modes}

The Rayleigh equation (3.4) is solved for three representative Mach numbers, $M=0.3$, 0.5 and 0.9 , by using a shooting method based on a fourth-order Runge-Kutta integrator. For each value of $b$, the neutral wavenumber $\alpha$, phase speed $c$ and hence frequency $\omega$ are obtained. For the convenience of comparing with experimental results, the usual Strouhal number $S t=2 R_{J} f^{*} / U_{J}=\omega / \pi$ is introduced, where $f^{*}$ is the physical frequency in Hertz.

Figure $1(a, b)$ shows the predicted phase speeds and the wavenumbers for a range of St. Also shown are the experimental data of Stromberg et al. (1980) and Suzuki $\&$ Colonius (2006) for cold jets. The former experiment was performed at a low Reynolds number $R=1800$, for which the initial shear layer was laminar. The latter was for a high-Reynolds-number $\left(R=3.5 \times 10^{5}\right)$ turbulent jet. By assuming that the flow signature at the outer edge of jet consists of predominantly linear instability modes (an assumption well justified, since nonlinear effects are confined within the critical layer), the phase and amplitude information of relevant modes was extracted by projecting the measured pressure signal to the local eigenfunctions of the compressible Rayleigh equation. The phase speed for each frequency shown was calculated from the phase evolution at the saturation point and thus can be appropriately compared with that of the neutral mode. A remarkably good agreement is noted. The Mach number appears to have very little effect. The phase speed of relatively high-frequency modes $(S t>0.4)$ is almost independent of $S t$.

The frequency of a neutral mode depends on the local shear-layer width $b$, which is a monotonically increasing function of the streamwise location. Relatively lowfrequency modes are of jet-column type; they correspond to large $b$. As $b$ decreases, high-frequency modes arise, and their wavelengths become progressively shorter 

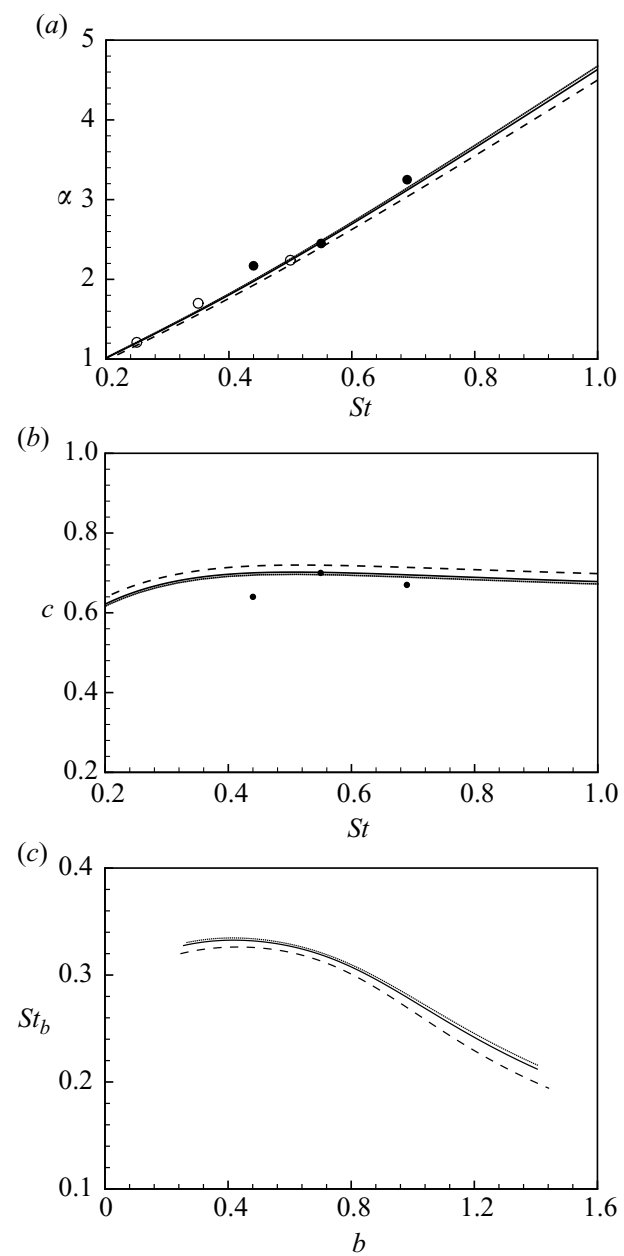

FigURE 1. (a) Wavenumbers $\alpha,(b)$ phase speed $c$ of neutral modes versus frequency $S t$ and $(c)$ Strouhal number $S t_{b} \equiv b S t$ versus $b$ : dotted lines, $M=0.3$; solid lines, $M=0.5$; dashed lines, $M=0.9$; $\bigcirc$, experiment of Suzuki \& Colonius $(2006)(M=0.5)$; $\bullet$, experiment of Stromberg et al. $(1980)(M=0.9)$.

and eventually acquire the character of shear-layer modes, so called because they concentrate in the thin shear layer centred at $r=1$. This change of character is demonstrated in figure $1(c)$, where $S t_{b} \equiv b S t$ is plotted against $b$. For $b<0.5, S t_{b}$ is almost independent of $b$, implying that the modes scale on the local shear-layer width $b$. Figure 2 displays the eigenfunctions of neutral modes with $S t=0.44$ and $S t=1.0$; the former features a jet-column mode, while the latter appears more like a shear-layer mode. Subsonic modes attenuate exponentially, as opposed to the algebraic decay of neutral supersonic modes (cf. Wu 2005).

\subsection{The nonlinear development of pairs of helical modes}

In order to assess the relative importance of non-parallelism, nonlinearity and nonequilibrium effects, we first solve the composite amplitude equation (4.5) numerically for the special case in which the temporal modulation is absent, i.e. $A$ is independent of $\hat{t}$. The spreading rate $\sigma_{s}$ is a key parameter, and the regimes in which one or more 


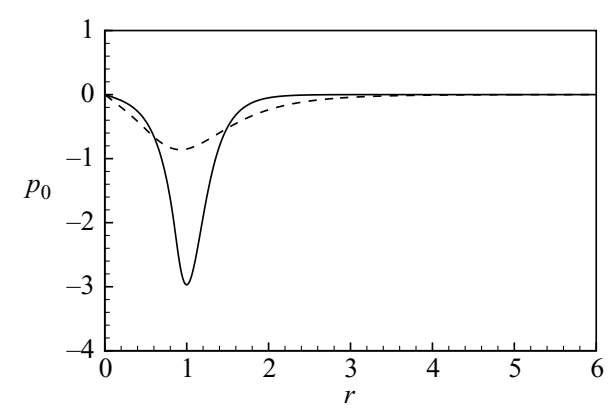

FIGURE 2. Eigenfunctions of subsonic instability modes at $M=0.9$ with $S t=1.0$ (solid line) and $S t=0.44$ (dashed line).

(a)

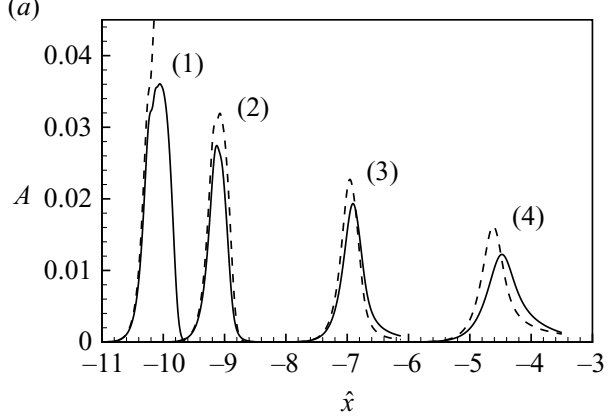

(b)

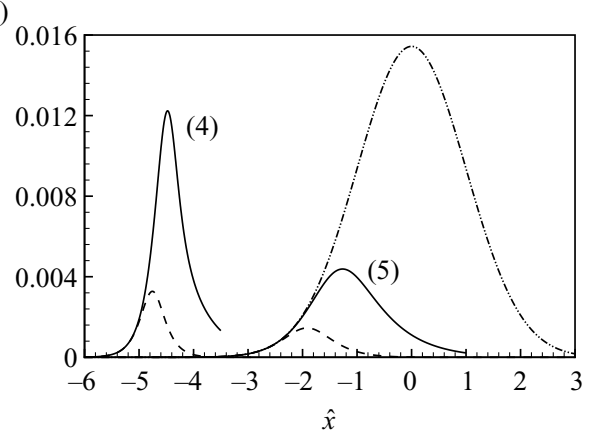

FIGURE 3. Nonlinear development of a subsonic instability mode with $S t=1$ at $M=0.9$ and $R=1800$ for an artificially small spreading rate $\sigma_{s}=1.36 \times 10^{-4}$. Curves (1)-(5) represent the result for 'initial amplitude' $a_{0}=2.68 \times 10^{21}, 6.71 \times 10^{16}, 8.18 \times 10^{8}, 5.10 \times 10^{2}, 1.54 \times 10^{-2}$. The dashed lines in $(a)$ and $(b)$ represent the predictions by the non-equilibrium parallel theory and equilibrium non-parallel theory respectively. The dashed-dotted line stands for the linear evolution.

of the effects dominate can be readily delineated for small spreading rates. We take $\sigma_{s}=1.36 \times 10^{-4}$, equivalent to a half-width spreading rate $b_{1 / 2}^{\prime}=5.5 \times 10^{-5}$, which is about three orders of magnitude smaller than typical realistic values. Figure 3 shows the amplitude development for different values of $a_{0}$. The predictions by the composite equation (4.5) are compared with those by its limiting forms (3.18) and (4.3).

Consistent with the result of Wu et al. (1993), in the parallel theory there exists a critical threshold initial amplitude, above which the amplitude develops a finitedistance singularity. The largest $a_{0}$ shown in figure 3 (curve (1)) is close to but slightly above the critical threshold, and so the singularity occurs in the solution given by the parallel theory. With non-parallelism included, the amplitude remains bounded. Although not shown here, it was found that as $a_{0}$ is increased further, the predictions by the two theories overlap to a great degree, and both feature a singularity, indicating that a critical threshold $a_{c}$ exists also in the non-parallel theory. For $a_{0}<a_{c}$, nonlinearity is found to have a stabilizing effect, causing the amplitude to attenuate at a location upstream of the linear neutral position. Overall, the larger the initial amplitude is, the earlier the nonlinearity comes into play, with the evolution occurring over a shorter scale. For these relatively large $a_{0}$ values both theories yield broadly similar results, suggesting that (3.18) is a valid leading-order approximation. 

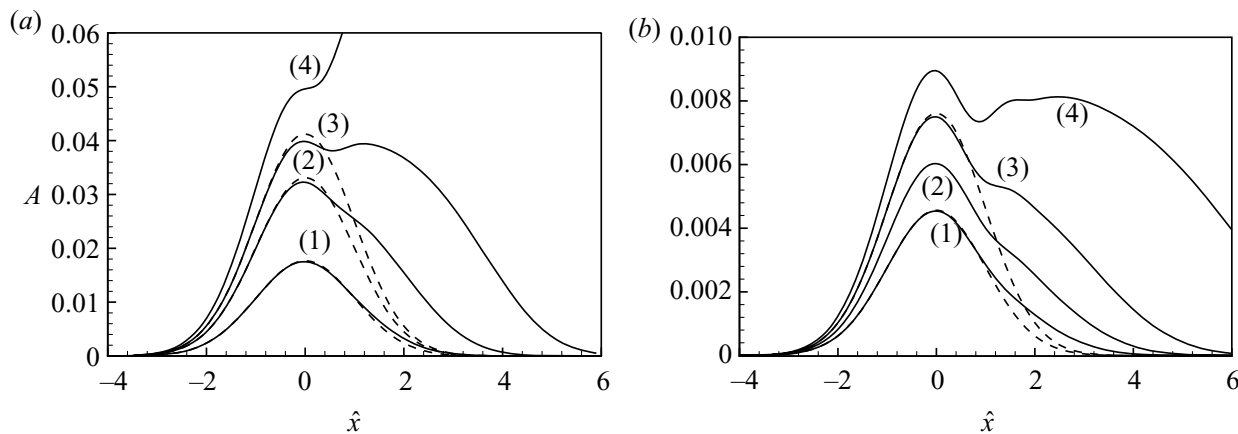

FiguRE 4. Nonlinear development of subsonic instability modes on a jet at $M=0.9$ and $R=1800$ with a realistic $\sigma_{s}=0.043$. (a) $S t=1$ and the initial amplitude $a_{0}=1.77 \times 10^{-2}$ (curve (1)), $3.31 \times 10^{-2}$ (curve (2)), $4.12 \times 10^{-2}$ (curve (3)) and $5.16 \times 10^{-2}$ (curve (4)). (b) $S t=0.44$ and $a_{0}=4.56 \times 10^{-3}$ (curve (1)), $6.09 \times 10^{-3}$ (curve (2)), $7.61 \times 10^{-3}$ (curve (3)) and $9.13 \times 10^{-3}$ (curve (4)). The dashed lines represent the linear evolution.

Non-parallelism nevertheless modifies the solution quantitatively; e.g. it increases the critical threshold $a_{c}$ required for the solution to remain bounded. On the other hand, as $a_{0}$ decreases, one might expect the instability modes to enter the equilibrium regime for which (4.3) holds. The prediction by the latter is displayed in figure $3(b)$. The comparison with the composite theory indicates that for the $\sigma_{s}$ considered, the equilibrium theory gives qualitatively similar results, but the quantitative agreement is rather poor: the attenuation occurs too early, and the maximum amplitude turns out to be too small. In conclusion, for the present extremely small $\sigma_{s}$, one may identify a parallel non-equilibrium regime (figure $3 a$ ) and non-parallel equilibrium regime (figure $3 b$ ), the key features of which can be captured by simplified amplitude equations (3.18) and (4.3), respectively. However, the composite evolution equation (4.5) has to be used for quantitative predictions.

Figure $4(a, b)$ shows the nonlinear development of subsonic modes on a jet with a realistic $\sigma_{s}=0.043$, extracted from a half-width spreading rate $b_{1 / 2}^{\prime}=0.017$, which is typical of the experimental conditions (e.g. Iqbal \& Thomas 2007). The Reynolds number is $R=1800$. As expected, there exists a critical $a_{c}$ such that for $a_{0}>a_{c}$, the amplitude terminates at a finite-distance singularity. Then it is necessary to construct a uniformly valid solution for $A$ by removing the singularity, which requires considering fully nonlinear Euler equations (Goldstein \& Choi 1989), before the emitted sound can be calculated. Waves of such larger amplitude and faster modulation would be a stronger source, but the radiation mechanism remains the same as elucidated here. In what follows, we shall focus on $a_{0}<a_{c}$, for which the amplitude remains bounded. For $\sigma_{s}$ values typical of experiments, it is no longer possible to identify any limiting regime, and instead all three factors now operate simultaneously. With the non-equilibrium effect included, nonlinearity has a slight stabilizing effect near the peak amplitude, after which its role is destabilizing, causing the disturbance to decay much more slowly than in the linear case. This differs even 'qualitatively' from the equilibrium nonlinearity, which would have been entirely stabilizing, since the Landau coefficient $l_{r}<0$. Such a slower-than-Gaussian decay was noted in the experiments of Suzuki \& Colonius (2006; see their figure 12a), and the overall growth, saturation and decay predicted for moderate $a_{0}$ are reminiscent of their measurements. The oscillatory feature exhibited by the amplitude at relatively large $a_{0}$, e.g. curve (3) in figure 4(a) and curves (3) and (4) in figure 4(b), qualitatively resembles some 


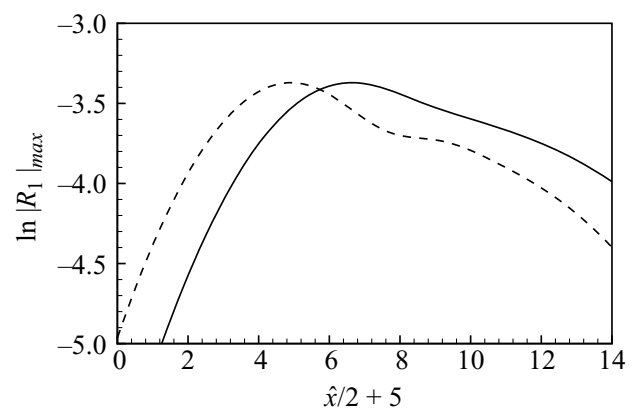

FIGURE 5. The axial distribution of the peak density fluctuation $R_{1} \equiv \epsilon R^{-1 / 3}\left(-\Theta_{1} / T_{c}^{2}\right.$ ) (solid line) of a mode with $S t=0.44$ and $a_{0}=7.61 \times 10^{-3}$ and comparison with the amplitude development (dashed line). The axial coordinate is the distance to the jet exit normalized by the diameter.

experimental observations made for helical modes in a turbulent jet, e.g. figure 5.26(b) of Ahuja et al. (1982).

It should be pointed that while the amplitude $A$ is proportional to the maximum pressure fluctuation, it does not have such a simple relation with the axial velocity or density fluctuations, which acquire their respective maximum magnitudes in the critical layer. Due to the non-equilibrium effect, both depend on the history of $A$, as indicated by (3.12). Figure 5 shows the axial development of the local maximum density fluctuation, which is proportional to the maximum axial-velocity fluctuation according to (3.12). The density evolution is contrasted with the amplitude $A$, renormalized such that its maximum equals that of the density. Their shapes are quite similar, but the density attenuates farther downstream, suggesting a strong non-equilibrium effect. It may be noted that the density development appears quite similar to the experimental result shown in figure 7 of Stromberg et al. (1980). Here for the convenience of comparison, the axial coordinate is taken to be the distance to the nozzle by assuming that the neutral position of the modes is located five diameters downstream.

In the absence of temporal modulation, the mean-flow distortion due to the mutual interaction between a pair of helical modes induces a steady mean-flow distortion, which is of interest in its own right and has been studied experimentally by several authors (Cohen \& Wygnanski 1987b; Long \& Petersen 1992).

We can write

$$
p_{m}(\tilde{x}, r)=J_{p}(\tilde{x}) q_{m}(r) .
$$

It follows from (3.29) that $q(r)$ satisfies

$$
\left.\begin{array}{c}
\left\{\frac{\partial^{2}}{\partial r^{2}}+\frac{1}{r} \frac{\partial}{\partial r}-\frac{4 m^{2}}{r^{2}}\right\} q_{m}+\left(\frac{\bar{T}^{\prime}}{\bar{T}}-\frac{2 \bar{U}^{\prime}}{\bar{U}}\right) \frac{\partial q_{m}}{\partial r}=0, \\
q_{m}\left(r_{c}^{+}\right)-q_{m}\left(r_{c}^{-}\right)=0, \quad q_{m}^{\prime}\left(r_{c}^{+}\right)-q_{m}^{\prime}\left(r_{c}^{-}\right)=1, \\
q_{m}^{\prime} \rightarrow \bar{U}^{2} \quad \text { as } \quad r \rightarrow \infty,
\end{array}\right\}
$$

where the behaviour of $q_{m}$ for large $r$ is derived by noting that for profile (5.1) and (5.2), $\bar{T}^{\prime} / T=0$ but $\bar{U}^{\prime} / \bar{U} \neq 0$, at infinity. 

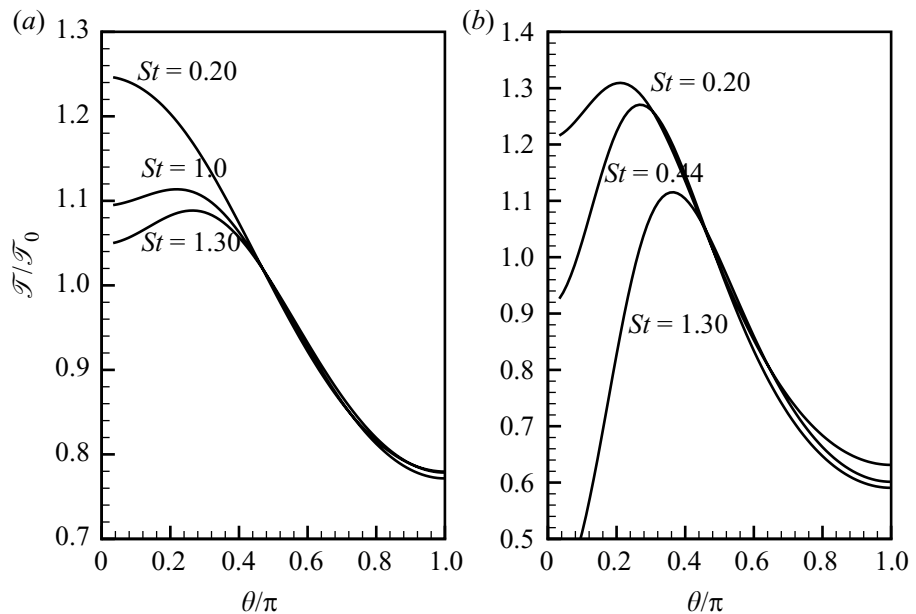

FIGURE 6. The normalized transfer function $\mathscr{T}(\theta ; S t) / \mathscr{T}_{0}$ versus $\theta$ at $(a) M=0.5$ and $(b)$ $M=0.9$.

In terms of $q_{m}$, the axial velocity $u_{m}$, which is of special interest, can be expressed as

$$
u_{m}(\tilde{x}, r)=c^{2} /\left(\bar{T}_{c} \bar{U}_{c}\right) \mathscr{J}_{u}(\tilde{x}) \frac{\bar{T} \bar{U}^{\prime}}{\bar{U}^{2}} q_{m}^{\prime}(r) .
$$

Matching with the corresponding critical-layer solution (3.13) yields

$$
\mathscr{D}_{m}=\left(q_{m}^{\prime}\left(r_{c}^{+}\right)-\frac{1}{2}\right) \mathscr{J}_{u}(\tilde{x}) .
$$

Using the multiplicative rule, we may construct a composite solution

$$
U_{m}^{(c)}=\left\{\begin{array}{l}
u_{m}(r) U_{m}\left(\left(r-r_{c}\right) R^{1 / 3}, \tilde{x}, \tilde{t}\right) / q_{m}^{\prime}\left(r_{c}^{+}\right) \quad \text { for } \quad r \geqslant r_{c}, \\
u_{m}(r) U_{m}\left(\left(r-r_{c}\right) R^{1 / 3}, \tilde{x}, \tilde{t}\right) / q_{m}^{\prime}\left(r_{c}^{-}\right) \text {for } r<r_{c},
\end{array}\right.
$$

which is uniformly valid for all $r$. The distorted axial flow, $\left(\bar{U}+\epsilon U_{m}^{(c)} \cos 2 m \phi\right)$, would become increasingly azimuthally dependent as it evolves downstream, and its contours in the $(r, \phi)$ plane would appear elliptic as observed in experiments (Cohen \& Wygnanski 1987b; Long \& Petersen 1992).

\subsection{The transfer function $\mathscr{T}(\theta ; S t)$}

The transfer function $\mathscr{T}(\theta ; S t)$ is determined by solving (3.52). Its dependence on $S t$ comes from the critical level $r_{c}$, which is a function of $\omega_{c}$, the carrier-wave frequency of the instability wavepacket. If we artificially set $\bar{U}^{\prime}=\bar{T}^{\prime}=0$, i.e. neglect the refraction of the background shear flow, (3.52) can be easily solved analytically to obtain

$$
\mathscr{T}_{0}=-r_{c}^{2 m+1} /(4 m),
$$

which is independent of $\theta$. The refraction effect can then be quantified by the transfer function $\mathscr{T}$ normalized by $\mathscr{T}_{0}$.

Figure $6(a, b)$ shows the variation of $\left|\mathscr{T}(\theta ; S t) / \mathscr{T}_{0}\right|$ with $\theta$ for $M=0.5$ and $M=0.9$. At moderate Mach number (e.g. $M=0.5), \mathscr{T}$ is a monotonic decreasing function of $\theta$ for carrier waves of relatively low frequencies. As $S t$ increases, a broad peak, centred at $\theta \approx \pi / 3$, starts to emerge. At $M=0.9, \mathscr{T}$ exhibits a peak for all $S t$ at an angle which 
increases with St. The peak becomes sharper at higher Mach numbers. An interesting and important fact is that $\left|\mathscr{T}(\theta ; S t) / \mathscr{T}_{0}\right|>1$ around the peak angle, indicating that the background shear flow acts as a 'soundboard' to amplify the emission along certain directions. Therefore, without taking into account the refraction effect, neither the directivity nor the intensity can be predicted correctly.

\subsection{The acoustic field of a linear wavepacket}

It is instructive to consider first the case in which the amplitude of a wavepacket is sufficiently small so that it remains linear during its entire evolution. Let $\widehat{A}(\bar{x}, \omega)$ denote the Fourier transform of $\bar{A}(\bar{x}, \bar{t})$. Then it follows that $\widehat{A}(\bar{x}, \omega)$ takes the form

$$
\widehat{A}(\bar{x}, \omega)=\widehat{A}_{0}(\omega) \exp \left(\frac{1}{2} \sigma \bar{x}^{2}-\mathrm{i} c_{g}^{-1} \omega \bar{x}\right)
$$

where $\widehat{A}_{0}(\omega)$ is the upstream spectrum of the wave envelope. As an example, we consider the special case in which $A_{0}$ is Gaussian

$$
\widehat{A}_{0}(\omega)=\sqrt{2 \pi} \hat{a}_{0} \mathrm{e}^{-d \omega^{2}}
$$

here $\hat{a}_{0}$ measures the overall intensity and $1 / \sqrt{d}$ characterizes the scaled spectral bandwidth, of the oncoming wavepacket. The wavepacket envelope in physical space is given by

$$
\bar{A}(\bar{x}, \bar{t})=\frac{\hat{a}_{0}}{\sqrt{2 d}} \exp \left(\frac{1}{2} \sigma \bar{x}^{2}-\left(\bar{x} / c_{g}-\bar{t}\right)^{2} /(4 d)\right),
$$

which is merely 'quasi-Gaussian', since its shape is deforming continuously when propagating downstream due to a complex group velocity $c_{g}$. Equation (5.6) indicates that the wavepacket decays to zero as $\bar{x} \rightarrow \pm \infty$. The wave envelope itself has an envelope,

$$
\overline{\bar{A}^{2}}=\hat{a}_{0}^{2} \sqrt{\frac{\pi}{2 d}} \exp \left(-\frac{1}{2} q \bar{x}^{2}\right)
$$

which is Gaussian, since

$$
q=-\left(\sigma+\sigma^{*}\right)+\left(1 / c_{g}-1 / c_{g}^{*}\right)^{2} /(4 d)>0
$$

is real. The axial extent in which the wavepacket is significant is measured by $1 / \sqrt{q}$.

It can be shown further that

$$
\begin{aligned}
\frac{\widehat{\mathscr{J}}_{p}(\bar{x}, \omega)}{\hat{a}_{0}^{2} j_{0}}=\sqrt{\frac{\pi}{2 d}} \int_{0}^{\infty} \exp \left\{-\frac{1}{2}\left[d \omega^{2}+\right.\right. & q(\bar{x}-\eta)^{2}+\mathrm{i} \omega\left(1 / c_{g}+1 / c_{g}^{*}\right) \\
& \left.\times(\bar{x}-\eta)]-\mathrm{i}(\omega / c) \eta-2 \bar{s} \eta^{3}\right\} \mathrm{d} \eta
\end{aligned}
$$

and that

$$
\frac{\widehat{\mathscr{J}}_{p}(k, \omega)}{\hat{a}_{0}^{2} j_{0}}=\frac{\pi}{\sqrt{q d}} \mathrm{e}^{-\left\{d \omega^{2}+\left(k+\omega\left(1 / c_{g}+1 / c_{g}^{*}\right) / 2\right)^{2} / q\right\} / 2} \mathscr{C}(k, \omega),
$$

where the factor $\mathscr{C}(k, \omega)$, defined as

$$
\mathscr{C}(k, \omega ; \bar{s})=\int_{0}^{\infty} \mathrm{e}^{-2 \bar{s} \eta^{3}-\mathrm{i}(k+\omega / c) \eta} \mathrm{d} \eta,
$$


represents the non-equilibrium effect. Inserting this into (3.53) yields the spectrum

$$
\begin{aligned}
\mathscr{I}(\omega ; \theta) /\left(\hat{a}_{0}^{4} j_{0}^{2}\right)=\omega^{4 m} \exp \left\{-\left[d+\frac{1}{q}\left(M_{a} \cos \theta\right.\right.\right. & \left.\left.\left.-\frac{1}{2}\left(1 / c_{g}+1 / c_{g}^{*}\right)\right)^{2}\right] \omega^{2}\right\} \\
& \times\left|\mathscr{C}\left(-M_{a} \omega \cos \theta, \omega\right)\right|^{2} .
\end{aligned}
$$

The directivity is given by

$\mathscr{D}(\theta)=\mathscr{T}(\theta)(\sin \theta)^{2 m}\left[\int_{-\infty}^{\infty} \omega^{4 m} \mathrm{e}^{-\left\{d+\left(M_{a} \cos \theta-\left(1 / c_{g}+1 / c_{g}^{*}\right) / 2\right)^{2} / q\right\} \omega^{2}}\left|\mathscr{C}\left(-M_{a} \omega \cos \theta, \omega\right)\right|^{2} \mathrm{~d} \omega\right]^{1 / 2}$.

In the equilibrium regime $\left(\bar{s}=s R^{1 / 2} \gg O(1)\right), \mathscr{C} \approx(1 / 3)(2 s)^{-1 / 3} \Gamma(1 / 3)$, independent of $\omega$, and so the expressions for $\mathscr{I}(\omega ; \theta)$ and $\mathscr{D}(\theta)$ reduce to

$$
\begin{gathered}
\mathscr{I}(\omega ; \theta) /\left(\hat{a}_{0}^{4} j_{0}^{2}\right)=\omega^{4 m} \exp \left\{-\left[d+\frac{1}{q}\left(M_{a} \cos \theta-\frac{1}{2}\left(1 / c_{g}+1 / c_{g}^{*}\right)\right)^{2}\right] \omega^{2}\right\} \mathscr{C}^{2}, \\
\mathscr{D}(\theta)=\mathscr{T}(\theta)(\sin \theta)^{2 m}\left\{d+\left(M_{a} \cos \theta-\frac{1}{2}\left(1 / c_{g}+1 / c_{g}^{*}\right)\right)^{2} / q\right\}^{-(m+1 / 4)} \mathscr{C}
\end{gathered}
$$

These results indicate that the acoustic field depends on all three parameters characterizing the envelope $\bar{A}$ and the physical source $\mathscr{J}_{p}$ : the group velocity $c_{g}$, frequency bandwidth $1 / \sqrt{d}$ and jet spreading rate $\sigma_{s}$. Since $c_{g}$ is complex, whose imaginary part $c_{g i}$ is typically one fourth of the real part $c_{g r}$, both $\bar{A}$ and $\mathscr{J}_{p}$ appear to be convecting downstream at speed $c_{g r}$. However, their profiles undergo continuous deformation because of a non-zero $c_{g i}$. As will be shown later, nonlinear effects cause further distortion of the wave envelope. The discussion above suggests that even in the present idealized situation, it would be too simplistic to characterize the source by a simple convection velocity.

We first consider a wavepacket with Strouhal number $S t=0.44$, which is chosen because it corresponds to the peak frequency of hydrodynamic fluctuations in the region in which the jet is nearly fully developed. The Reynolds number $R=1800$ is taken to be the same as in the experiments of Stromberg et al. (1980). Instead of $1 / \sqrt{d}$, the unscaled frequency bandwidth

$$
\Delta \omega \equiv 1 / \sqrt{R d}
$$

will be used as a parameter because the latter is easily related to the carrier-wave frequency $\omega_{c}=\pi S t$. For example, for the wavepacket with $S t=0.44$ to be considered, we take $\Delta \omega=0.3$ and 0.5 , which correspond to bandwidths of about $\omega_{c} / 5$ and $\omega_{c} / 4$ respectively. Figure $7(a, b)$ displays the directivity of the emitted sound and its spectrum at $\theta=90^{\circ}$, predicted by (5.11) and (5.10) respectively for $\Delta \omega=0.5$. In order to assess the non-equilibrium effect, the prediction by formulae (5.12) and (5.13) is also included, which is a valid approximation when the critical layer is equilibrium, i.e. when $s \gg 1$. At this low Reynolds number, the results are quantitatively similar. The acoustic field features a single-lobed pattern, indicating that the sound concentrates in a beam which makes an angle of about $\theta_{p}=46^{\circ}$ to the jet axis. It consists of a band of low-frequency components. At $90^{\circ}$, the spectrum peaks at $S t_{p}=0.06 \sim 0.08$. Nevertheless, neglecting the non-equilibrium effect leads to an appreciable overprediction of the sound intensity, as well as a slightly smaller inclination angle of the beam. The difference becomes much more substantial for high Reynolds numbers in 

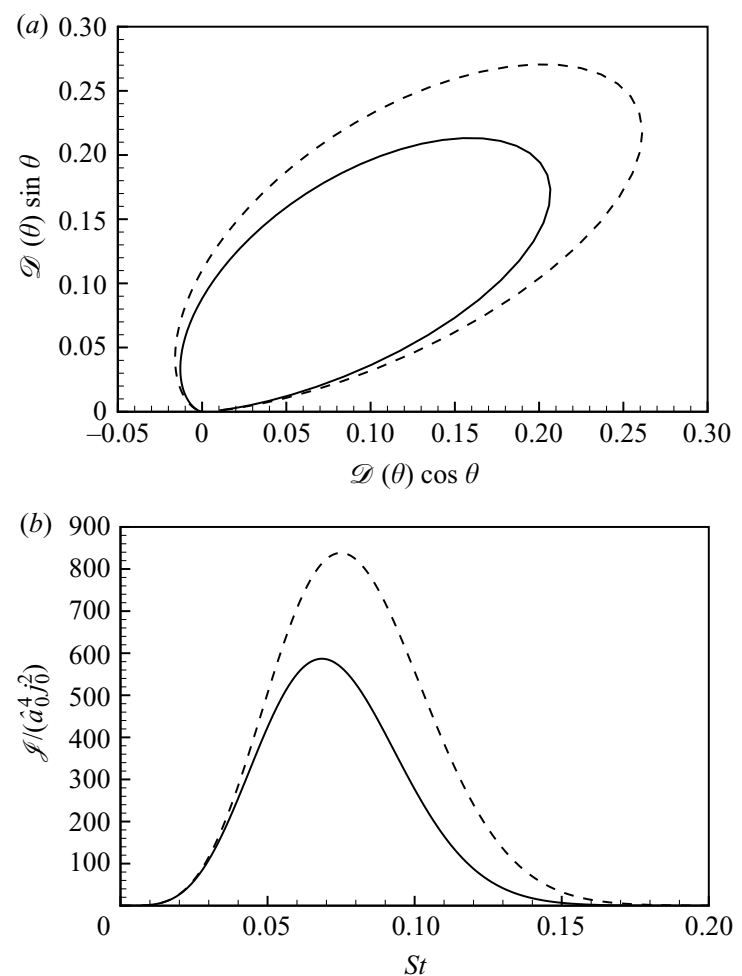

Figure 7. ( $a$ ) The directivity and $(b)$ the spectrum at $\theta=90^{\circ}$ of the acoustic field radiated by a linear wavepacket with $S t=0.44$ and $\Delta \omega=0.5$. Solid lines: non-equilibrium result; dashed lines: equilibrium result. Reynolds number $R=1800$ and $\sigma_{s}=0.043$.

typical experiments (e.g. $\left.R=O\left(10^{6}\right)\right)$. In the following, only (5.10) and (5.11) will be used.

Figure $8(a, b)$ shows the effects of $\sigma_{s}$ (the jet spreading rate) and $\Delta \omega$ (the bandwidth of the spectrum). When $\sigma_{s}$ is halved, $\theta_{p}$ is reduced from $54^{\circ}$ to $50^{\circ}$, while $S t_{p}$ drops from 0.07 to 0.053 , and meanwhile the acoustic intensity is more than doubled. Increasing $\Delta \omega$ (from 0.3 to 0.5 ) reduces $\theta_{p}$ (from $54^{\circ}$ to $46^{\circ}$ ), but its primary effect is to enhance the acoustic field as expected, while the peak frequency $S t_{p}$ is hardly altered. Also shown in the figure is the spectrum at $\theta=\theta_{p}=46^{\circ}$. In comparison with the $90^{\circ}$ spectrum, a broader peak centred at a higher $S t$ is observed.

The Reynolds number influences the $\mathscr{D}(\theta)$ and $\mathscr{I}(\omega, \pi / 2)$ spectra via $s$ (see (5.9) and (5.10)), and its effect is demonstrated in figure $9(a, b)$. At $R=5.4 \times 10^{5}$, the directivity remains similar to that in the $R=1800$ case albeit with a slightly smaller $\theta_{p}=50^{\circ}$. A notable change is that the spectrum exhibits double peaks, and the acoustic energy shifts to lower-frequency components.

The salient features of the acoustic field appear to be robust and are qualitatively consistent with experimental measurements. There are however noticeable quantitative differences: the predicted $\theta_{p}$ (about $60^{\circ}$ ) is larger and $S t_{p}$ (about 0.07) is smaller the than typical experimental data $\theta_{p} \approx 30^{\circ}$ and $S t_{p} \approx 0.2$. It should be noted that in experiments $\theta$ is defined with the origin being taken at the nozzle, while in our theory $\theta$ is defined with the origin taken to be at the neutral position of the instability mode under consideration. On taking into account the fact that the latter is usually about 

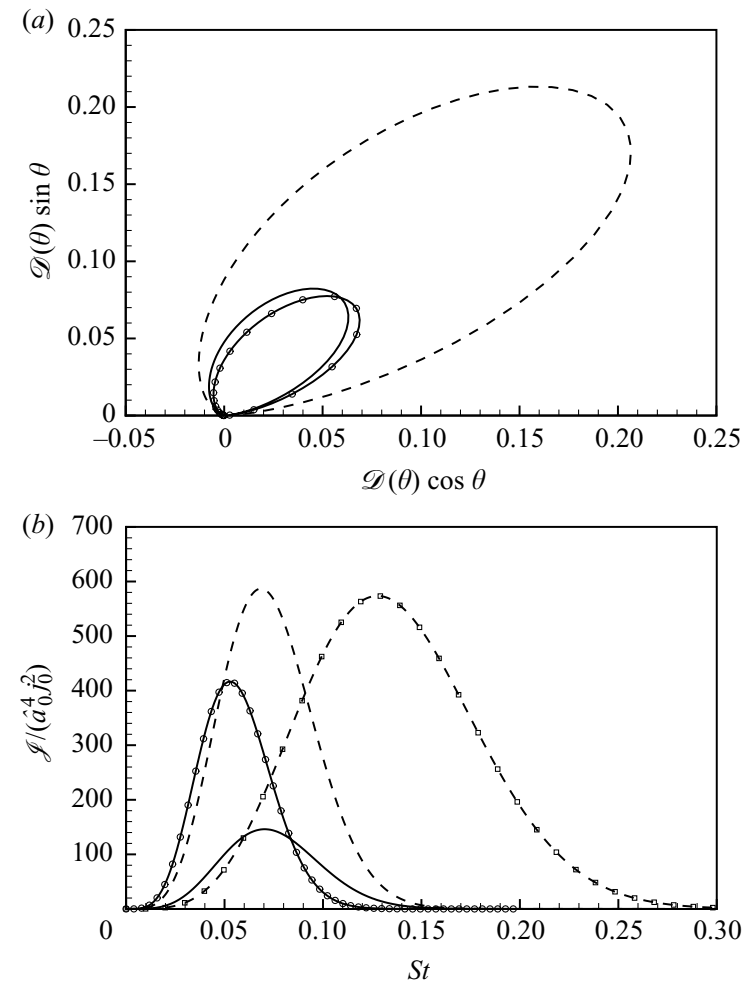

FIGURE 8 . The $(a)$ directivity and $(b)$ spectrum of the acoustic field radiated by a linear wavepacket with $S t=0.44$ at $R=1800$. Solid lines: $\sigma_{s}=0.043$ and $\Delta \omega=0.3$; solid lines with symbols: $\sigma_{s}=0.0215$ and $\Delta \omega=0.3$; dashed lines without symbols: $\sigma_{s}=0.043$ and $\Delta \omega=0.5$. In $(b)$, the dashed line with symbols represents the spectrum at $\theta=46^{\circ}\left(\sigma_{s}=0.043\right.$ and $\left.\Delta \omega=0.5\right)$.

4-6D downstream of the nozzle, the experimental value (measured at a distance of $30 \mathrm{D}$; Stromberg et al. 1980) is converted to $\theta_{p} \approx 40^{\circ}$, which is somewhat closer to the prediction, but appreciable difference remains.

While a wavepacket with $S t=0.44$ is representative of the most energetic hydrodynamic fluctuations in the region towards the end of the potential core, it does not necessarily contribute the dominant noise (Freund 2001). Next, we consider the acoustic field radiated by a wavepacket with $S t=1.0$, as a representative of relatively high-frequency components, which are likely to be present in the upstream region before the end of the potential core.

In figure $10(a, b)$, the directivity and spectrum for selected values of $\sigma_{s}$ and $\Delta \omega(0.5$ and 0.8$)$ are displayed; here $\Delta=0.5$ and 0.8 correspond to bandwidths of about $\omega_{c} / 6$ and $\omega_{c} / 4$ respectively. The characteristics of the beam appears almost identical to that in the $S t=0.44$ case (cf. figure 8 ). The spectral peak shifts to higher frequencies as might be expected. It is worth noting that while $\theta_{p}=55^{\circ}$ is larger than the experimental value, the peak frequency in the spectrum in this direction is $S t_{p}=0.22$, which is well within the range observed in experiments.

Increasing the Reynolds number to $R=2.7 \times 10^{5}$ does not substantially alter the qualitative features of the directivity and spectrum, as is shown in figure $11(a, b)$. However, at high Reynolds numbers, a wavepacket with the same amplitude emits much more intense sound than at low Reynolds numbers (cf. figures $10 b$ and $11 b$ ). 

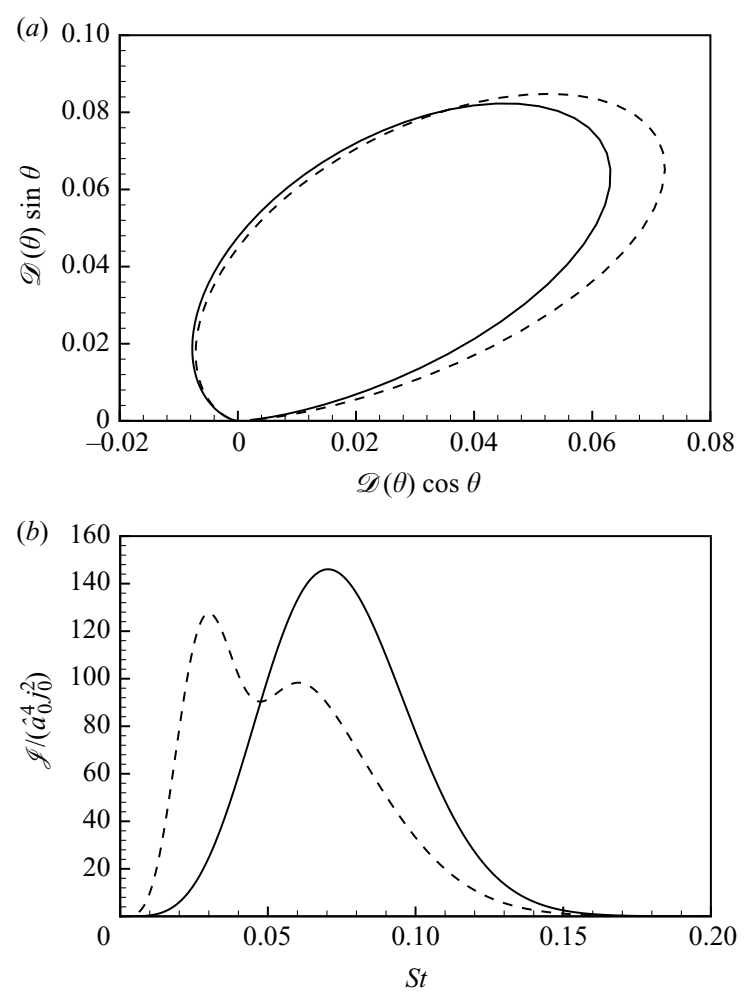

FIGURE 9. Effect of the Reynolds number on the $(a)$ directivity and $(b)$ spectrum of the acoustic field radiated by a linear wavepacket with $S t=0.44, \Delta \omega=0.3$ and $\sigma_{s}=0.043$. Solid lines: $R=1800$; dashed lines: $R=2.7 \times 10^{5}$.

\subsection{The acoustic field of a nonlinear wavepacket}

In order to compute the acoustic field of a nonlinear wavepacket, we solve the amplitude equation (4.5) by taking the Fourier transform with respect to $\bar{t}$ (cf. Wu 2005). The amplitude in spectral space is inverted to evaluate the nonlinear term in physical space, which is then Fourier transformed back to spectral space. For $\bar{x} \rightarrow-\infty$, the nonlinear term is negligible so that (5.5) can be used as the initial condition', imposed at a large negative $x_{0}$. An Adams-Moulton (implicit) method of sixth-order accuracy was employed to march the solution downstream.

Figure 12(a) shows the envelope development of a wavepacket with $S t=1.0$ for three different values of initial amplitude $a_{0}$. In the linear limit, the envelope follows a Gaussian distribution (5.7). As $a_{0}$ increases, the envelope deviates from this shape due to the nonlinear effect. Interestingly, nonlinearity appears 'dormant' during the growing phase of the wavepacket and asserts its influence only in the decaying phase, causing the envelope to decay at a slower rate than that in the linear limit. Double peaks appear for relatively large $a_{0}$. The axial profiles overall look quite similar to those in the non-modulated case (cf. figure 4).

The directivity $\mathscr{D}(\theta)$ of the acoustic field emitted by the wavepacket is shown in figure $12(b)$ for different sizes of initial amplitude $a_{0}=R^{-7 / 6} \hat{a}_{0} / \sqrt{2 d}$. As $a_{0}$ increases, the lobed beam is tilted slightly away from the jet axis, but its gross feature is not significantly altered by nonlinearity. Since $\mathscr{D}(\theta)$, or the distance of the tip of the lobe to the origin, measures the 'efficiency' of emission, the increase of the overall lobe 

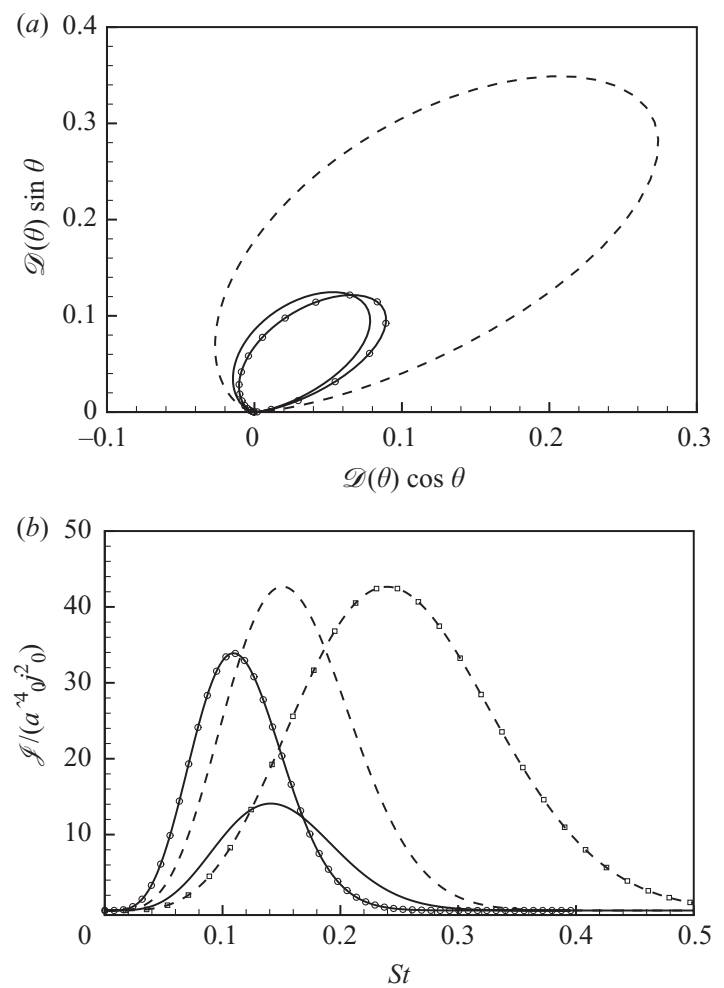

FiguRE 10 . The $(a)$ directivity and $(b)$ spectrum of the acoustic field radiated by a linear wavepacket with $S t=1.0$ at $R=1800$. Solid lines: $\sigma_{s}=0.043$ and $\Delta \omega=0.5$; solid lines with symbols: $\sigma_{s}=0.0215$ and $\Delta \omega=0.5$; dashed lines without symbols: $\sigma_{s}=0.043$ and $\Delta \omega=0.8$. In $(b)$, the dashed line with symbols represents the spectrum at $\theta=55^{\circ}\left(\sigma_{s}=0.043\right.$ and $\left.\Delta \omega=0.8\right)$.

size with $a_{0}$ indicates that as a wavepacket envelope is distorted by the nonlinear effect, it emits stronger sound. Meanwhile, the spectrum of the acoustic field becomes broader, as shown in figure $12(c)$. For the largest $a_{0}$ considered, the frequency band is approximately in the range $0.1-0.3$, broadly consistent with the experimentally observed range of the acoustic spectral peak. Though not shown here, a similar effect of nonlinearity is observed for $S t=0.44$.

\section{Conclusions and further discussions}

In this paper, we have investigated sound waves emitted by a wavepacket consisting of a pair of interacting helical instability waves with nearly identical frequencies. Based on relevant previous theoretical work on nonlinear instability, a composite amplitude equation, which takes into account the effects of non-equilibrium, nonlinearity and nonparallelism, was proposed to describe the entire growth-attenuation-decay cycle of the wavepacket. The streaming effect of the wave interaction generates a strong slowly breathing, azimuthally dependent mean flow distortion. An analysis of its far-field asymptotic behaviour shows that it acts as the dominant emitter of lowfrequency sound waves. The latter was determined by a matched-asymptotic expansion procedure. Parametric studies pertaining to relevant experimental conditions indicate that the acoustic field is characterized by a single-lobed directivity pattern beamed at an angle of about $45^{\circ}-60^{\circ}$ to the jet axis and a broadband spectrum centred at 

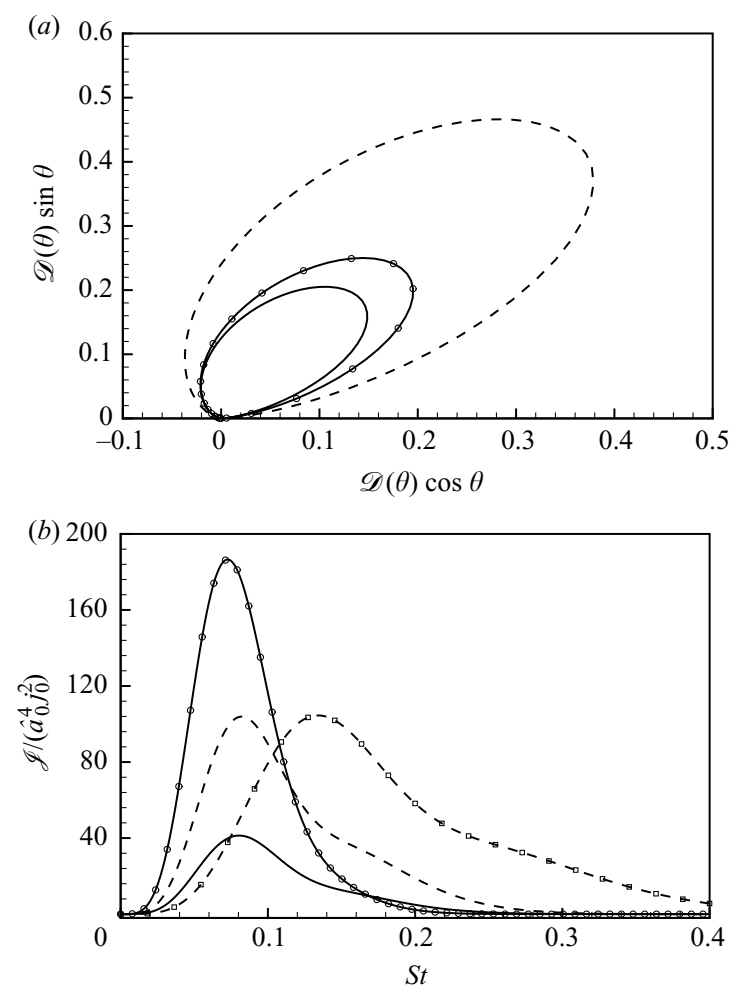

Figure 11. The $(a)$ directivity and $(b)$ spectrum of a linear wavepacket with $S t=1.0$ at Reynolds number $R=2.7 \times 10^{5}$. Solid lines: $\sigma_{s}=0.043$ and $\Delta \omega=0.5$; solid lines with symbols: $\sigma_{s}=0.0215$ and $\Delta \omega=0.5$; dashed lines without symbols: $\sigma_{s}=0.043$ and $\Delta \omega=0.8$. In $(b)$, the dashed line with symbols represents the spectrum at $\theta_{p}=53^{\circ}\left(\sigma_{s}=0.043\right.$ and $\left.\Delta \omega=0.8\right)$.

a Strouhal number $S t \approx 0.07-0.2$. A wavepacket evolving nonlinearly was found to radiate more efficiently, and the spectrum of the noise broadens considerably while its directivity remains almost unaltered.

In the present first-principles theory, the dominant noise 'emitter' (i.e. the streaming induced 'mean field') is identified in an unambiguous manner by analysing the largedistance behaviour of the hydrodynamic field, without making an ad hoc attribution of source. The theory therefore describes the precise physical process of sound generation, which is found to involve an 'inverse energy cascade', a non-compact source and the refraction effect of the background shear flow. The streaming-acoustics mechanism revealed here is likely to be of fundamental importance for understanding noise generation in subsonic and moderate-Mach-number supersonic jets, just as the Mach-wave radiation is for high-Mach-number jet noise, where dominant structures propagate supersonically. In particular, the present mechanism provides a possible explanation for the amplification of the low-frequency portion of noise by pure-tone excitation, since forcing a jet at a single frequency induces a response in the form of a narrowband wavepacket (e.g. Stromberg et al. 1980), which then emits broadband low-frequency sound waves.

As was remarked earlier, the present theory was prompted by the observations made by Gamard et al. (2004). Further evidence supporting the theory can be inferred from the experiments of Panda \& Seasholtz (2002), Panda, Seasholtz \& Elam 

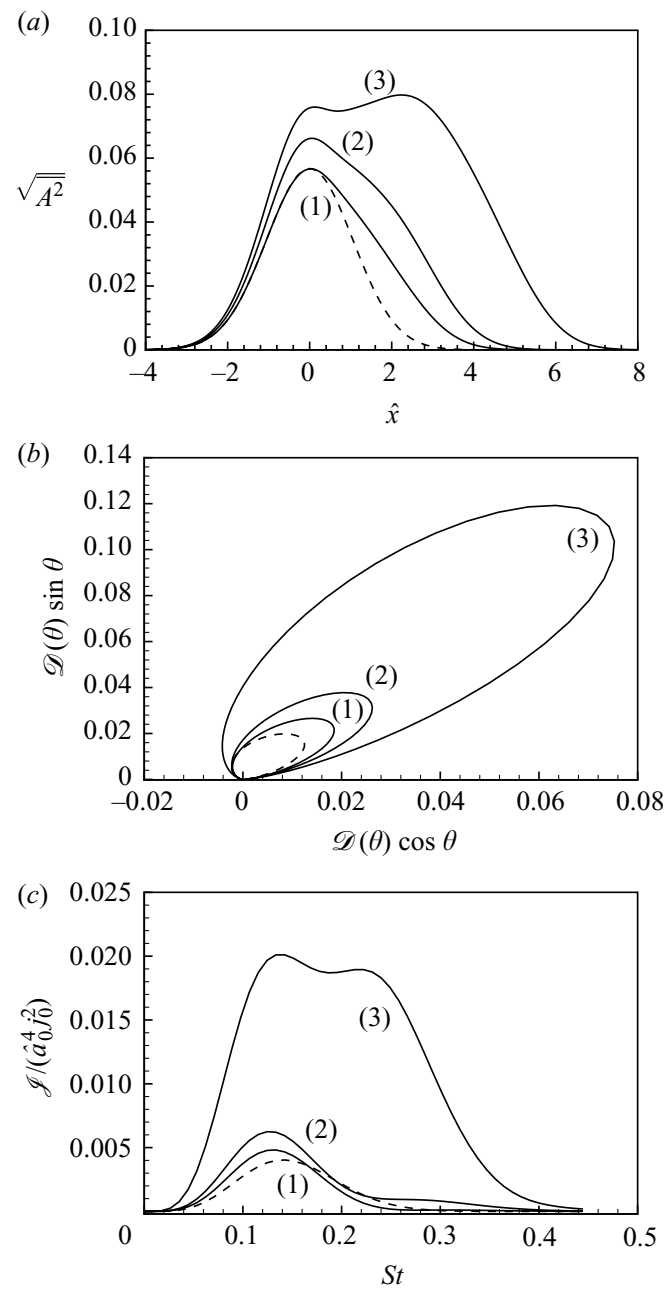

FIGURE 12. The nonlinear development of a wavepacket (with $S t=1.0, \Delta \omega=0.5$ ) and its acoustic field. (a) $\sqrt{\overline{A^{2}}}$ versus $\hat{x}$. (b) Directivity pattern. (c) Spectrum at $\theta=90^{\circ}$. Initial amplitude $a_{0}=2.53 \times 10^{-2}$ (curve (1)), $a_{0}=2.95 \times 10^{-2}$ (curve (2)), $a_{0}=3.38 \times 10^{-2}$ (curve (3)). The dashed lines represent the result for a linear wavepacket with $a_{0}=2.53 \times 10^{-2}$. Reynolds number $R=1800$ and $\sigma_{s}=0.043$.

(2005) and Panda (2007), who found that the far-field sound correlates strongly with the low-frequency $(S t=0.05-0.2)$ components of the density fluctuation within the jet, while there is little correlation with high-frequency $(S t>0.4)$ components. The low-frequency density fluctuation seems most likely to be the signature of the mean density distortion $\rho_{m}$ induced by the nonlinear interaction. Since $\rho_{m}$ is proportional to the axial-velocity distortion $u_{m}$ (3.13) (Leib \& Lee 1995), the strong correlation with $\rho_{m}$ implies therefore a strong correlation with $u_{m}$, which is of course entirely consistent with, and indeed expected by, our theory.

Our theory was formulated and predictions were made for a well-defined realizable disturbance, and so they can be validated by numerical simulations and/or experiments in which the assumed instability modes $(m= \pm 1)$ are excited in a controlled manner. In the laminar case, direct calculations of the acoustic far 
field should be feasible, at least for relatively low Reynolds numbers. For high Reynolds numbers, to reduce computational costs one might have to take a 'twostage' approach, in which the hydrodynamic near field is resolved first, and the far field is subsequently predicted either $(a)$ by using an acoustic analogy equation forced by (distributed or concentrated) sources in the form of Reynolds stress or quadrupoles or $(b)$ by propagating the relevant element of the near field via solving a linear acoustic equation. Approach $(a)$, though most popular and successful in many occasions, suffers from several well-acknowledged problems such as a rather ambiguous distinction of the source and propagation effects and sensitivity to the error in sources leading to spurious noise. To these, we would like to add a further note of caution that this approach may not always adequately describe the 'inverse energy cascade' as explained in $\$ 3.4$. In contrast, approach $(b)$ would appear conceptually clear and natural, and its implementation may be aided by the understanding gained from the present study. Since the slowly modulated standing wave in the azimuthal direction with $m=2$ has been identified as the emitter, one may extract this specific flow signature from the hydrodynamic solution and then propagate it to the far field to obtain the correct solution for the acoustics. Usually, this method requires the computation domain to be sufficiently large so that the numerical solution at the outer edge of the domain has acquired the large-radial-distance asymptotic behaviour. This stringent requirement, however, may not be necessary in the present problem because based on the fact that the emitter is driven locally within the critical layer, the local solution in its immediate vicinity may be continued to the entire field by solving a 'homogeneous' Lilley's equation. Note that the forcing imposed is in terms of the slowly breathing mean-flow distortion rather than in the form of Reynolds stresses (or quadrupoles), and so the procedure described above is subtly different from usual acoustic analogy (e.g. Sandham \& Salgado 2008), in that Lilley's equation now merely governs propagation but is no longer relied upon for the inverse energy cascade.

At low speeds, it was possible in laboratory to introduce helical modes with specified frequencies and azimuthal wavenumbers and follow their nonlinear interaction (Cohen \& Wygnanski 1987b; Long \& Petersen 1992; Corke \& Kusek 1993). A verification of the mechanism proposed in the present paper would require extending these controlled excitation techniques to the compressible regime to generate $m= \pm 1$ modes and to carry out simultaneous acoustic measurements. An additional requirement is the capacity of appropriately modulating each mode, so as to control the frequency content of the resulting breathing 'mean flow', which determines the emitted acoustic field. Intriguingly, in the incompressible regime strong fluctuations in the the low-frequency band (see e.g. figure 16 of Corke \& Kusek 1993) were observed. It would be interesting to examine the role of these components in noise generation at high speeds and their possible connections with the mechanism presented here. We hope that the present theoretical work would spur such experiments.

It is noted that while the gross features of the acoustic field predicted by the present study mimic experimental observations qualitatively, considerable quantitative differences exist: the angle between the direction of maximum emission and the jet axis is too large, and the results cannot account for sound waves in the relatively high-frequency $(0.3<S t<4)$ portion of the acoustic spectrum. The discrepancy and inadequacy may be due in part to the fact that the analysis is for a wavepacket consisting of just two modulated helical modes, which radiate sound waves with azimuthal wavenumber $m= \pm 2$ only, while in reality noise is contributed by broadband instability waves (and also by small-scale turbulence). Interaction of 
multiple modes is probably most significant close to the jet nozzle because coexisting axisymmetric and helical shear-layer modes in that region have comparable growth rates and magnitudes (e.g. Cohen \& Wygnanski 1987a). An investigation of their nonlinear development and the associated acoustic radiation is currently in progress. In particular, the interaction of multiple modes can emit sound waves with azimuthal numbers $m=0$ and $m= \pm 1$, which tend to concentrate along the directions that make shallow angles with the jet axis. Moreover, the shear-layer modes have much higher frequencies $(S t \approx 4)$, and so the beating interaction among them may contribute to the medium-frequency $(0.4<S t<0.8$, say) portion of the emitted noise. It may be expected that once this extra contribution is accounted for, the discrepancies with experiments would be reduced.

As we explained in the introduction, the present theory was primarily formulated for a laminar jet. It was nevertheless found to capture some qualitative features of turbulent jet noise. The demonstrated relevance is underpinned by two facts. Firstly, the physical mechanism described is general and robust: provided that a wave-wave interaction generates a breathing mean flow, low-frequency sound waves are emitted. Secondly, coherent wave-like structures in turbulent jets closely resemble instability modes, as suggested by overwhelming experimental measurements. In the literature on coherent structures, analyses involving instabilities modes, such as the present one, are sometimes viewed as being for the mean field of a turbulent flow. Unfortunately, it is impossible to justify this viewpoint on a rigorous mathematical or a fundamental physical footing, for the very notion of 'instability of a turbulent flow' is open to serious question. Inevitably, at the current stage one has to approach coherent structures on an empirical basis. Characterizing them in terms of instability modes, thereby constructing noise prediction models, appears to be a potentially fruitful empirical framework. Even at this the empirical level, a number of effects, unique to turbulent flows, remain to be investigated. First, while it has been established (Suzuki \& Colonius 2006) that coherent structures are well approximated by linear eigenmodes in an extensive radial region, the detailed dynamics in the nonlinear region and its impact on the overall evolution is not understood. We believe that the nonlinear critical-layer theory, developed for laminar flows, is broadly relevant. The new factor to be considered for turbulent jets is the influence of small-scale turbulence on coherent structures. If an eddy-viscosity type of model is used, the net effect would be a decreased equivalent Reynolds number, and so the qualitative behaviour would remain similar. Further theoretical and experimental work is needed to test this speculation. Second, coherent structures in a turbulent flow have a continuum of spectrum. It has been observed that a small number of low-order POD or eigenmodes may capture a significant fraction of the 'hydrodynamic' kinetic energy. However, how they interact and radiate sound is yet to be fully understood. Furthermore, since sound emission depends sensitively on the flow field, it is not at all certain that the same set of modes would form an adequate basis for predicting the majority of the 'acoustic' energy in the low-frequency portion of the spectrum. Thirdly, small-scale turbulence is generally viewed as a distinctive source emitting relatively high-frequency noise, but a first-principles description of the precise mechanism does not actually exist; despite that acoustic analogy approach has been regarded by many as a general framework. In summary, an ultimate model capable of quantitatively accurate predictions relies on further progress on the identification of a (hopefully small) set of large-scale modes responsible for noise generation, on parameterization of the effect of smallscale turbulence on coherent modes, on quantitative descriptions of their nonlinear interaction and the radiation process and finally on parameterization of the noise of 
small-scale turbulence (which may continue to rely upon acoustic analogy aided by presumably universal statistic properties of small-scale motions).

The bulk of this work was carried out when XW was visiting the LadHyx, Ecole Polytechnique Paris, during March-April, 2007. It is a pleasure to thank Professor Jean-Marc Chomaz and Dr Joseph Nicholas for discussions and hospitality. Helpful comments and suggestions from the anonymous referees are gratefully acknowledged.

\section{Appendix. The limiting forms of (4.5) and (4.6)}

Here we demonstrate that the composite evolution system (4.5) and (4.6) describes both the non-parallel equilibrium and parallel non-equilibrium regimes in the sense that it reduces to the respective limit forms for suitable sizes of $\bar{a}_{0}$.

For $\bar{a}_{0}=O(1),(4.5)$ can readily be reduced to (4.3) by the same procedure as in appendix B of Wu et al. (1993), namely by performing the substitution $\xi \rightarrow R^{-1 / 4} \xi$ (and $\zeta \rightarrow R^{-1 / 6} \zeta$ ) and taking the limit $R \gg 1$.

On the other hand, when $\bar{a}_{0} \gg O(1)$ we write

$$
\bar{x}=-\bar{\Delta}+x^{\dagger} / \bar{\Delta}, \quad \bar{t}=t^{\dagger} / \bar{\Delta}, \quad \bar{A}=\bar{\Delta} A^{\dagger},
$$

where $\bar{\Delta}>0$ is chosen to be

$$
\bar{\Delta} \mathrm{e}^{-\frac{1}{2} \sigma_{r} \bar{\Delta}^{2}}=\left|\bar{a}_{0}\right|
$$

so that $\bar{\Delta} \gg 1$. The above relation determines the location at which the disturbance first enters the nonlinear regime in terms of the initial amplitude: the larger the amplitude, the earlier the nonlinear evolution commences. Inserting (A 1) into (4.5) shows that

$$
\begin{aligned}
\frac{\partial A^{\dagger}}{\partial x^{\dagger}}+c_{g}^{-1} \frac{\partial A^{\dagger}}{\partial t^{\dagger}}= & \sigma\left(-1+x^{\dagger} / \bar{\Delta}^{2}\right) A^{\dagger}+\left(l / \bar{\Delta}^{4}\right) R^{2 / 3} \int_{0}^{\infty} \int_{0}^{\infty} K\left(\xi, \eta ; s^{\dagger}\right) A^{\dagger}\left(x^{\dagger}-\xi, t^{\dagger}-\xi / c\right) \\
& \times A^{\dagger}\left(x^{\dagger}-\xi-\eta, t^{\dagger}-\xi / c-\eta / c\right) A^{\dagger *}\left(x^{\dagger}-2 \xi-\eta, t^{\dagger}-2 \xi / c-\eta / c\right) \mathrm{d} \xi \mathrm{d} \eta,
\end{aligned}
$$

where $s^{\dagger}=s R^{1 / 2} / \bar{\Delta}^{3}$. This indicates that the non-equilibrium effect is of secondary importance provided $\bar{\Delta} \ll O\left(R^{1 / 6}\right)$. The non-equilibrium regime corresponds to the distinguished scaling $\bar{\Delta}=R^{1 / 6} \Delta$ with $\Delta=O(1)$, which is realized for

$$
\left|\bar{a}_{0}\right|=R^{1 / 6} \exp \left(-\frac{1}{2} \sigma_{r} \Delta^{2} R^{1 / 3}\right) \Delta .
$$

On noting that $\tilde{x}=x^{\dagger} / \Delta=O(1)$ and that the non-parallel effect $x^{\dagger} / \bar{\Delta}^{2} \ll O(1)$ is a higher-order correction, (A 3) reduces to (3.18). The initial condition can be rewritten as $\tilde{A} \rightarrow \mathrm{e}^{\sigma \Delta \tilde{x}} \tilde{A}_{l}\left(\tilde{x}-c_{g} \tilde{t}\right)$, the same as $(3.20)$.

\section{REFERENCES}

Ahuja, K. K., Lepicovsky, J., Tam, C. K. W., Morris, P. J. \& Burrin, R. H. (Lockhead-Georgia Co.) 1982 Tone-excited jet: theory and experiments. NASA-CR-3538. National Aeronautics and Space Administration.

Akylas, T. R. \& Toplosky, N. 1986 The sound field of a Tollmien-Schlichting wave. Phys. Fluids 29 (3), 685-689.

Arndt, R. E. A., Long, D. F. \& Glauser, M. N. 1997 The proper orthogonal decomposition of pressure fluctuations surrounding a turbulent jet. J. Fluid Mech. 340, 1-33. 
BALSA, T. F. 1975 Fluid shielding of low frequency convected sources by arbitrary jets. J. Fluid Mech. 70, 17-36.

Bechert, D. W. \& Pfizenmaier, E. 1975 On the amplification of broadband jet noise by a pure tone excitation. J. Sound Vib. 43, 581-587.

Bechert, D. W. \& Pfizenmaier, E. 1977 Amplification of jet noise by a higher-mode acoustical excitation. AIAA J. 15, 1268-1271.

Bishop, K. A., Ffowcs Williams, J. E. \& Smith, W. 1971 On the noise sources of the unsuppressed high-speed jet. J. Fluid Mech. 50, 21-31.

Bogey, C., Barre, S., Fleury, V., Bailly, C. \& Juve, D. 2007 Experimental study of the spectral properties of near-field and far-field jet noise. Intl J. Aeroacoust. 6 (2), 73-92.

Bradshaw, P., Ferriss, D. H. \& Johnson, R. F. 1964 Turbulence in the noise-producing region of a circular jet. J. Fluid Mech. 19, 591-624.

Bridges, J. \& Hussain, F. 1992 Direct evaluation of aeroacoustic theory in a jet. J. Fluid Mech. 240, 469-501.

Chimonas, G. \& Grant, J. R. 1984 Shear excitation of atmospheric gravity waves: upscale scattering from Kelvin-Helmholtz waves. J. Atmos. Sci. 41, 2278-2288.

Chan, Y. Y. 1974 Spiral waves in turbulent jets. Phys. Fluids 17, 46-53.

Churilov, S. M. \& Shukhman, I. G. 1994 Nonlinear spatial evolution of helical disturbances to an axial jet. J. Fluid Mech. 281, 371-402.

Citriniti, J. H. \& GeORGE, W. K. 2000 Reconstruction of the global velocity field in the axisymmetric mixing layer utilizing the proper orthogonal decomposition. J. Fluid Mech. 418, 137-166.

Corke, T. C. \& KuseK, S. M. 1993 Resonance in axisymmetric jets with controlled helical-mode input. J. Fluid Mech. 249, 07-336.

Crighton, D. G. \& Gaster, M. 1976 Stability of slowly divergent jet flow. J. Fluid Mech. 77, 397-413.

Crighton, D. G. \& Huerre, P. 1990 Shear-layer pressure fluctuations and superdirective acoustic sources. J. Fluid Mech. 220, 355-368.

Cohen, J. \& Wygnanski, I. 1987 a The evolution of instabilities in the axisymmetric jet. Part 1. The linear growth of disturbances near the nozzle. J. Fluid Mech. 176, 191-219.

Cohen, J. \& Wygnanski, I. $1987 b$ The evolution of instabilities in the axisymmetric jet. Part 2. The flow resulting from the interaction between two waves. J. Fluid Mech. 176, 221-235.

Cowley, S. J. \& WU, X. 1994 Asymptotic approaches to transition modelling. In Progress in transition modelling, AGARD Rep. 793. Advisory Group for Aerospace Research and Development.

Crow, S. C. 1972 Acoustic gain of a turbulent jet. Paper IE.6. In American Physical Society Meeting, University of Colorado, Boulder CO.

Crow, S. C. \& Champagne, F. H. 1971 Orderly structure in jet turbulence. J. Fluid Mech. 48, $547-591$.

Freund, J. B. 2001 Noise sources in a low-Reynolds-number turbulent jet at Mach 0.9. J. Fluid Mech. 438, 277-305.

FritTS, D. C. 1982 Shear excitation of atmospheric gravity waves. J. Atmos. Sci. 39, 1936-1952.

Gamard, S., Jung, D. \& George, W. K. 2004 Downstream evolution of the most energetic modes in a turbulent axisymmetric jet at high Reynolds number. Part 2. The far-field region. J. Fluid Mech. 514, 205-230.

Goldstein, M. E. 1975 The low-frequency sound from multipole sources in axisymmetric shear flows, with applications to jet noise. J. Fluid Mech. 70, 595-604.

Goldstein, M. E. 1976 The low-frequency sound from multipole sources in axisymmetric shear flows. Part 2. J. Fluid Mech. 75, 17-28.

Goldstein, M. E. 1984 Sound generation and upstream influence due to instability wave interacting with non-uniform mean flows. J. Fluid Mech. 149, 161-177.

Goldstein, M. E. 1995 The role of nonlinear critical layers in boundary-layer transition. Phil. Trans. R. Soc. Lond. A352, 425-442.

Goldstein, M. E. \& CHOI, S.-W. 1989 Nonlinear evolution of interacting oblique waves on twodimensional shear layers. J. Fluid Mech. 207, 97-120.

Goldstein, M. E. \& LeiB, S. J. 1989 Nonlinear evolution of oblique waves on compressible shear layers. J. Fluid Mech. 207, 73-96. 
Goldstein, M. E. \& Leib, S. J. 2008 The aeroacoustics of slowly diverging supersonic jets. J. Fluid Mech. 600, 291-337.

Haj-Hariri, H. \& Akylas, T. R. 1986 Sound radiation by instability wavepackets in a boundary layer. Stud. Appl. Math. 75, 57-76.

Hileman, J. I., Thurow, B. S., Caraballo, E. J. \& Samimy, M. 2005 Large-scale structure evolution and sound emission in high-speed jets: real-time visualization with simultaneous acoustic measurements. J. Fluid Mech. 544, 277-307.

Hussain, A. K. M. F. \& Hasan, M. A. Z. 1985 Turbulence suppression in free turbulent shear flows under controlled excitation. Part 2. Jet-noise reduction. J. Fluid Mech. 150, 159-168.

Hussain, A. K. M. F. \& Zaman, K. B. M. Q. 1980 Vortex pairing in a circular jet under controlled excitation. Part 2. Coherent structure dynamics. J. Fluid Mech. 101, 493-544.

IqBaL, M. O. \& Thomas, F. O. 2007 Coherent structure in a turbulent jet via a vector implementation of the proper orthogonal decomposition. J. Fluid Mech. 571, 281-326.

Jung, D., Gamard, S. \& George, W. K. 2004 Downstream evolution of the most energetic modes in a turbulent axisymmetric jet at high Reynolds number. Part 1. The near-field region. J. Fluid Mech. 514, 173-204.

Khavaran, A. \& Bridges, J. 2004 Modeling of turbulence generated noise in jet. AIAA Paper 2004-2983. American Institute of Aeronautics and Astronautics.

Kibens, V. 1980 Discrete noise spectrum generated by an acoustically excited jet. AIAA J. 18, 434-441.

Laufer, J. \& Yen, T. 1983 Noise generation by a low-Mach-number jet. J. Fluid Mech. 134, 1-31.

LEIB, S. J. 1991 Nonlinear evolution of subsonic and supersonic disturbances on a compressible free shear layer. J. Fluid Mech. 224, 551-578.

LEIB, S. J. \& LEE, S. S. 1995 Nonlinear evolution of a pair of oblique instability waves in a supersonic boundary layer. J. Fluid Mech. 282, 339-371.

Long, T. A. \& Petersen, R. A. 1992 Controlled interactions in a forced axisymmetric jet. Part 1. The distortion of the mean flow. J. Fluid Mech. 235, 37-55.

Lush, P. A. 1971 Measurements of subsonic jet noise and comparison with theory. J. Fluid Mech. 46, 477-500.

Michalke, A. 1971 Instabilitat eines kompressiblen runden Freistrahls unter Beruchksichtigung des Einflusses der Strahlgrenz-schichtdicke. Z. Flugwiss. 19, 319.

Mitchell, B. E., Lele, S. K. \& Moin, P. 1999 Direct computation of the sound generated by vortex pairing in an axisymmetric jet. J. Fluid Mech. 383, 113-142.

Mollo-Christensen, E. L., Kolpin, M. A. \& Martuccelli, J. R. 1964 Experiments on jet flows and jet noise far-field spectra and directivity patterns. J. Fluid Mech. 18, 285-301.

Moore, C. J. 1977 The role of shear-layer instability waves in jet exhaust noise. J. Fluid Mech. 80, 321-367.

PANDA, J. 2007 Experimental investigation of turbulent density fluctuations and noise generation from heated jets. J. Fluid Mech. 591, 73-96.

Panda, J. \& Seasholtz, R. G. 2002 Experimental investigation of density fluctuations in high-speed jets and correlation with generated noise. J. Fluid Mech. 450, 97-130.

Panda, J., Seasholtz, R. G. \& Elam, K. A. 2005 Investigation of noise sources in high-speed jets via correlation measurements. J. Fluid Mech. 537, 349-385.

Sandham, N. D., Morfey, C. L. \& Hu, Z. W. 2006 Nonlinear mechanisms of sound generation in a perturbed parallel jet flow. J. Fluid Mech. 565, 1-23.

Sandham, N. D. \& Salgado, A. 2008 Nonlinear interaction model of subsonic jet noise. Phil. Trans. R. Soc. Lond. 366 (1876), 2745-2760.

SCINOCCA, J. F. \& FoRD, R. 2000 The nonlinear forcing of large-scale internal gravity waves by stratified shear instability. J. Atmos. Sci. 57, 653-672.

Strange, P. J. R. \& Crighton, D. G. 1983 Spinning modes on axisymmetric jets. Part 1. J. Fluid Mech. 134, 231-245.

Stromberg. J. L., McLaughlin, D. K. \& Troutt, T. R. 1980 Flow field and acoustic properties of a Mach number 0.9 jet at low Reynolds number. J. Sound Vib. 72 (2), 159-176.

SUZUKI, T. \& Colonius, T. 2006 Instability waves in a subsonic round jet detected using a near-field phased microphone array. J. Fluid Mech. 565, 197-226.

TAM, C. K. W. 1971 Directional acoustic radiation from a supersonic jet generated by shear layer instability. J. Fluid Mech. 46, 757-768. 
TAM, C. K. W. 1995 Supersonic jet noise. Annu. Rev. Fluid Mech. 27, 17-43.

TAM, C. K. W. \& Burton, D. E. 1984 Sound generated by instability waves of supersonic flow. Part 2. Axisymmetric jets. J. Fluid Mech. 138, 273-295.

TAM, C. K. W. \& MorRis, P. J. 1980 The radiation of sound by the instability waves of a compressible plane turbulent shear layer. J. Fluid Mech. 98, 349-381.

WU, X. 2005 Mach wave radiation of nonlinearly evolving supersonic instability modes in shear layers. J. Fluid Mech. 523, 121-159.

WU, X. \& HogG, L. 2006 Acoustic radiation of Tollmien-Schlichting waves as they undergo rapid distortion. J. Fluid Mech. 550, 307-347.

Wu, X., LeE, S. S. \& Cowley, S. J. 1993 On the weakly nonlinear three-dimensional instability of shear flows to pairs of oblique waves: the Stokes layer as a paradigm. J. Fluid Mech. 253, 681-721.

Zaman, K. B. M. Q. 1985 Far-field noise of a subsonic jet under controlled excitation. J. Fluid Mech. 152, 83-111.

Zaman, K. B. M. Q. 1986 Flow field and near and far sound field of a subsonic jet. J. Sound Vib. 106, $1-16$.

Zaman, K. B. M. Q. \& Hussain, A. K. M. F. 1980 Vortex pairing in a circular jet under controlled excitation. Part 1. General jet response. J. Fluid Mech. 101, 449-491.

Zaman, K. B. M. Q. \& Hussain, A. K. M. F. 1984 Natural large-scale structures in the axisymmetric mixing layer. J. Fluid Mech. 138, 325-351. 